\title{
La preuve dans les sciences sociales
}

\section{Giovanni Busino}

\section{OpenEdition}

\section{Journals}

Édition électronique

URL : http://journals.openedition.org/ress/377

DOI : $10.4000 /$ ress.377

ISSN : 1663-4446

\section{Éditeur}

Librairie Droz

\section{Édition imprimée}

Date de publication : 10 décembre 2003

Pagination : 11-61

ISBN : 2-600-00913-2

ISSN : 0048-8046

Référence électronique

Giovanni Busino, "La preuve dans les sciences sociales », Revue européenne des sciences sociales [En ligne], XLI-128 | 2003, mis en ligne le 11 novembre 2009, consulté le 30 avril 2019. URL : http:// journals.openedition.org/ress/377; DOI : 10.4000/ress.377 


\section{Giovanni BUSINO}

\section{LA PREUVE DANS LES SCIENCES SOCIALES}

SOMMAIRE: 1. Prologue. - 2. Bref rappel historique de la notion. -3 . La médecine. -4 . Le droit. -5 . La préhistoire et l'archéologie. -6 . La géographie et la démographie. - 7. L'économie. - 8. La sociologie. - 9. L'histoire. 10. Epilogue.

\section{PROLOGUE}

Reprenant une analyse wébérienne, Jean-Claude Passeron ${ }^{1}$ fonde son épistémologie des sciences sociales sur le fait que le chercheur y est confronté à une variabilité, dans le temps et dans l'espace, des corrélations entre les éléments constitutifs des configurations historiques singulières qu'il observe, et non à la répétition régulière des «faits» instituant une relation entre certains éléments d'une situation d'ensemble, qui pourraient alors être isolés et comparés toutes choses étant égales par ailleurs. Les «faits» historiques sont ainsi marqués par la singularité de leur insertion dans un «contexte» auquel ils adhèrent significativement et causalement, à la différence des «faits » naturels constitués de «traits » dont la sélection et la reconstruction par l'expérimentation peuvent donner lieu à l'observation de répétitions régulières, susceptibles de fonder la formulation de régularités ou de «lois» scientifiques; ils différent plus encore des entités ou des opérations logiques qui permettent, dans les sciences formelles, des « démonstrations », au sens strict du terme.

De fait, observe Jean-Claude Passeron, il n'a jamais pu se constituer, dans les sciences sociales, de langage protocolaire unifié de la description des états du monde; les théories y ont toujours revêtu des formes argumentatives concur-

* Je dois exprimer ma gratitude et mes remerciements à Jean-Blaise Grize, à Pierre Livet et à JeanClaude Passeron pour leurs précieuses remarques grâce auxquelles certaines formulations de ce texte ont pu être améliorées. L'aide de Victor Monnier m'a permis de prendre connaissance des controverses juridiques, de consulter les publications de la Société Jean Bodin et de mettre à jour plusieurs notes bibliographiques. A lui aussi je dis mes sentiments amicaux et reconnaissants.

J.-Cl. Passeron, La forme des preuves dans les sciences historiques, «Revue européenne des sciences sociales », XXXIX, 2001, n. 120, pp. 31-76. Cette question avait été déjà posée dans le livre du même auteur, Le raisonnement sociologique. L'espace non-poppérien du raisonnement naturel, Paris, Nathan, 1991, et dans divers articles publiés dans Le modèle et l'enquête. Les usages du principe de rationalité dans les sciences sociales, Paris, Ed. de l'EHESS, 1995, et dans Le modèle et le récit, Paris, Ed. de la MSH, 2001. 
rentes, du fait même qu'elles dosent différemment des méthodologies de la preuve hétérogènes entre elles, ne pouvant que les associer dans un raisonnement naturel pour les faire «converger» sémantiquement. Les descriptions y sont inévitablement «inachevées», toujours susceptibles d'être enrichies ou reprises dans un autre lexique et une autre grammaire; les procédures probatoires y différent donc, par leur définition épistémologique du «dire vraie», de celles qui ont cours dans les disciplines formelles ou expérimentales. Les langages protocolaires qui peuvent être utilisés dans les sciences sociales sont donc locaux, sectoriels et contextualisés, parce que les paramètres et les variables ne peuvent y être isolés et contrôlés, ni comme les définitions d'une logique formelle ou d'une axiomatique, ni comme les concepts expérimentaux permettant la description entièrement définie d'une série d'observations: ils ne se prêtent à la quantification que par l'autonomisation momentanée de leur sens opératoire par rapport à leur sens contextuel.

Dans la descriptions du «cours du monde historique», que Passeron distingue ainsi du «cours historique du monde $»^{2}$, la démonstration formelle de forme logiquement ou mathématiquement "pure », le raisonnement expérimental à visée nomologique, de même que - et pour la même raison - le recours à une herméneutique universelle, ne constituent pas le ressort principal de l'administration de la preuve ou de la construction des évidences. Ces démarches occupent, certes, une place, irremplaçable, dans les opérations d'un ensemble auquel elles doivent être restituées pour prendre tout leur sens empirique, c'est-à-dire pour formuler des assertions scientifiques - susceptibles d'être dotés de plus ou moins de «véridicité »-sur le monde réel des déroulements historiques.

Suffit-il, à l'encontre de cette thèse, de faire valoir, comme on le fait depuis longtemps, que les «objets » de la plupart des sciences de la matière ou de la vie, comme la géophysique, la paléontologie, la cosmologie et la biologie ${ }^{3}$, sont, elles aussi, inscrites dans le temps et dans l'espace, qu'elles sont les produits d'enchấnements complexes, d'événements divers et singuliers, non reproductibles, et dont l'élucidation ou l'explication dépendent des variables contextuelles? Faut-il rappeler, en outre, que le contexte n'est jamais constant lors de l'interprétation des corrélations, des variations concomitantes et d'autres analogies, qu'il délimite le domaine d'application des théories explicatives et les rend provisoires? En outre, les énoncés, dans n'importe quel système symbolique, véhiculent des abstractions dissociées, des renvois directes ou connotatifs à des éléments concrets de l'expérience sociale. Dès lors l'allongement des questionnements, des interprétations, les intégrations des concepts dans des réseaux d'énoncés abstraits et l'absence de

Sur cette distinction cf. J.-C. Passeron, Statistique et dynamique. Caractériser, expliquer, comprendre le changement, «Revue européenne des sciences sociales», XXXVI, 1998, n. 110, pp. 199- 263, mais également du même Logique et schématique dans l'argumentation des sciences sociales, Ibid., XXXV, 1997, n. 107, pp. 169-196.

Dans ces disciplines, on remonte, à partir d'une certaine connaissance actuelle, vers un événement singulier antérieur et sans analogue aujourd'hui. Le «big bang» en est l'exemple le plus typique. On pourrait également citer, entre autres, la «déduction anthropique» grâce à laquelle on déduit l'existence de l'homme de certaines propriétés de la matière et de certains états antérieurs de l'Univers. 
contraintes sont inéluctables, même dans les pragmatiques herméneutiques s'opposant à l'explication ${ }^{4}$.

Les débats autour de la «preuve» (méthode standard, démonstration, expérimentation, validation empirique, formation des hypothèses, sélection des déterminations susceptibles de réplication, vérification), de l' «explication », de la «compréhension » et de la nature de la scientificité des disciplines sociales, continuent à se situer au centre des recherches de l'épistémologie contemporaine des sciences ${ }^{5}$.

Bien que l'atomisme opérationnaliste transforme une relation idéelle entre concepts en une relation empirique entre indicateurs; bien que les doctrines de la preuve conditionnelle et partielle doivent faire les comptes avec la double adéquation (significative et causale, compréhensive et expérimentale); bien que la théorie de la preuve par la cohérence ne puisse jamais être dégagée des apories de la circularité logique, néanmoins l'une ou l'autre de ces trois approches restent généralement fort prisées par la majorité des «social scientists ». Ces derniers sont tous persuadés que les conditions d'établissement des assertions valides suffisent pour déterminer des observables bien formés alors que nous travaillons sur des modèles et des hypothèses, sur des «objets » que l'on croit être en dehors de nous et qu'en fait ne sont que nos représentations.

Il est vrai que ces trois approches ont été ébranlées récemment à la fois par les chercheurs qui inscrivent la preuve dans la logique de la réflexivité, de l'indexicalité et de l'accountability et par les partisans des philosophies postmodernistes, du relativisme anthropologique et des sociologies des sciences ${ }^{6}$. Néanmoins elles

4 J.-Cl. Gardin, Les embarras du naturel, «Revue européenne des sciences sociales», XXXIV, 1996, n. 103, pp. 215-228, mais aussi ce que cet auteur a écrit dans Modèles et Récits, in Epistémologie des sciences sociales, sous la direction de J.-M.Berthelot, Paris, Puf, 2001, pp. 407-454. Des objections de la même nature ont été formulées par des «sociologistes » comme P. Bourdieu, des « individualistes méthodologiques » comme R. Boudon, des marxistes comme L. Althusser ou N. Poulantzas, des sociologues holistes comme L. A. Coser, des chercheurs en sciences dures comme J.-P. Changeux. Des psychologues expérimentaux, qui espéraient mettre à la base de toutes les sciences historiques les «lois» universelle d'une science «nomologique» des comportements humains, et des historiens mécanistes ou marxistes ont été adressées des objections analogues à Max Weber. Celui-ci les a analysées notamment dans l'essai de 1904 Die «Objektivität» Sozialwissenschaftlicher und sozialpolitischer Erkenntnis (à présent in Gesammlte Aufsätze

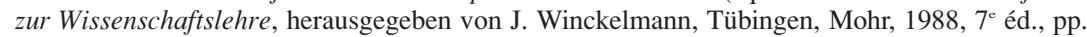
146-214) où il écrit que, même lorsqu'elles prennent pour objet des phénomènes indissociables de la singularité du déroulement temporel où on les observe, les sciences de la nature ne sont pas dans la même situation méthodologique que les sciences de la société face à la tâche d'en faire comprendre la temporalité historique. Les sciences sociales sont, en effet, dépourvues - en tant que sciences de l'homme aspirant à expliquer in devenir - d'un savoir nomologique constitué, semblable à celui dont disposent les sciences nomologiques qui ont déjà établi et réussi à relier au sein d'un paradigme théorique, des «lois» d'interdépendance entre les phénomènes (psycho. chimiques par, exemple), lois universellement valables.

5 En sciences humaines les problèmes d'intelligibilité, de persuasion, d'objectivité, de correspondance des propositions aux «faits», de vérification (confirmation) des énoncés factuels, se mélangent et se confondent avec les questions de la plausibilité, de l'assentiment, de la conviction dans la charge de la preuve. Les distinctions entre les techniques de la preuve et la logique de la preuve ou de l'interprétation y sont à peine distinguables. Cf. F.Gil, Preuves, Paris, Aubier, 1988 et G.-G. Granger, La vérification, Paris, Odile Jacob, 1992.

${ }^{6} \quad$ R. Ogien, Les causes et les raisons. Philosophie analytique et sciences humaines, Nîmes, J. Chambon, 1995. Voir également du même Philosophie des sciences sociales, in Epistémologie 
constituent toujours le patrimoine commun des chercheurs, lesquels lui attribuent une importance indiscutable. Chez ces chercheurs l'induction (le passage par l'expérience immédiate et sensible pour aller du particulier au général) reste un point ferme, indubitable, en dépit du fait que sa légitimation et ses fondements demeurent fragiles, sinon incertains, que sa nature est fondamentalement heuristique ${ }^{7}$. Cela tient au fait qu'il n'a pas été possible jusqu'ici de définir la réalité sociale ainsi que les entités, les propriétés et les mécanismes qui la composent. Les tentatives pour consolider la doctrine du naturalisme social ${ }^{8}$, les travaux sur les états mentaux en tant que dispositifs physiques déterminés, sur les croyances collectives et individuelles réduites à des fonctionnements cérébraux, les philosophies et les sociologies de l'esprit et de l'action, renvoient tantôt à un monisme ontologique fondamental tantôt à des dispositions ontologiques disparates et variées ${ }^{9}$. Dès lors les descriptions multiples, la pluralité des jeux de langage, les faits actuels et virtuels constituent, pour les chercheurs, des contraintes difficiles à surmonter. Les travaux de Talcott Parsons, de Jürgen Habermas, de James Coleman, de Pierre Bourdieu, d'Antony Giddens, de Raymond Boudon et de tant d'autres, plus ou moins basés sur la présupposition que les «objets sociaux » sont aussi réels que les «objets physiques » alors qu'ils sont toujours des « objets » d'un modèle, - ces travaux sont des exemples éloquents.

Sans doute, tous ces débats sont fondamentaux, enrichissants, nécessaires. Assurément ils foisonneront, au fil des années à venir, encore davantage. Toutefois je voudrais les laisser de côté, ne pas me déterminer à leur sujet, être libre de questionner rhapsodiquement les pratiques effectives des régimes de la preuve, d'ébaucher un inventaire succinct des dénotations et des connotations qui leur sont attribuées dans le champ sémantique des sciences humaines. Ici il m'importe surtout de comprendre comment dans la pratique quotidienne s'opère la justification, comment nos disciplines fabriquent et puis utilisent les "preuves », comment la communauté scientifique les légitime ou les rejette. Enfin, je voudrais déchiffrer si en raison de la grande diversité des modes de production et de réception des preuves dans les sciences sociales, celles-ci peuvent aspirer à la dignité de science $^{10}$.

des sciences sociales, op. cit., pp. 521-575 et P. Livet, Action et cognition en sciences sociales, Ibid., pp. 269-316.

Les critiques de Popper (une connaissance est inductive si elle émane intégralement du monde extérieur, mais cela implique une attitude totalement passive de nos capacités cognitives) n'ont pas sapé la doctrine de J.S. Mill, A System of Logic Ratiocinative and Inductive, livre III, chapitres I, II, III, IV : «Toute inférence et, conséquemment, toute preuve et toute découverte de vérité non évidentes de soi consistent en inductions et en interprétations d'inductions; [...] toute notre connaissance non intuitive provient exclusivement de cette source. En conséquence, la question de la nature de l'induction et des conditions qui la rendent légitime est incontestablement la question fondamentale de la logique, celle qui embrasse toutes les autres.»

${ }^{8}$ Pour en savoir davantage lire de L. Quéré, La validité de l'argument naturaliste en sciences sociales, in L'argumentation. Preuve et persuasion, Paris, Ed. de l'EHESS, 2002, pp. 93-117.

$9 \quad$ Une publication récente fixe, de manière magistrale, les contours de cette problématique. Voir P. Livet et R. Ogien (éds.), L'enquête ontologique : du mode d'existence des objets sociaux, Paris, Ed. de l'EHESS, 2000, coll. «Raisons pratiques, n. $11 »$.

10 Il y a quelques tentatives déjà faites à propos de l'œuvre d'un chercheur particulier. Par exemple, les preuves apportées par Margaret Mead dans ses enquêtes sur les mœurs et la sexualité en 


\section{BREF RAPPEL HISTORIQUE DE LA NOTION}

Aussi loin que l'on remonte dans le temps, la définition générale de la preuve renvoie aux procédures ayant pour but l'établissement de la connaissance valide. La nature de ces procédures reste hétérogène ou estompée. Tantôt il s'agit de montrer ad oculos une chose, de présenter un fait ou d'établir la vérité d'une assertion tantôt il s'agit d'exhiber un document, de rapporter un témoignage, d'effectuer une induction. Dans certaines circonstances la preuve est une présentation et dans d'autres un raisonnement syllogistique (déductif, enthymème). La notion a une extension plus grande que celle de démonstration expérimentale. En effet, toutes les preuves ne sont pas des démonstrations bien que celles-ci constituent toujours des preuves ${ }^{11}$.

Aristote élabore la première classification et distingue la preuve en non artificielle, ne dépendant pas de l'art de l'orateur (lois, contrats, témoignages, écrits, tortures, serments) et en artificielle (syllogismes, arguments, exemples, étymologies, etc.). Le domaine de la preuve est celui du doute et du probable. Pour cette raison il fait du syllogisme le producteur de la preuve et de la preuve la source du savoir $^{12}$. L'indice, ou signe apparent, est caractérisé par la probabilité. Il distingué l'eikos (le semblable) du payradeigma (modèle, exemple), du semeion (signe, marque distinctive) et du tekmerion (signe de reconnaissance, inconfutable, à la base de l'argumentation persuasive $)^{13}$. L'enthymème rapproche la rhétorique à la

Océanie, ont été rudement contestées par divers anthropologues. Tel débat a été reconstruit par S. Tcherkézoff, Le mythe occidental de la sexualité polynésienne, 1928-1999. Margaret Mead, Derek Freeman et Samoa, Paris, Puf, 2001. Très utile est le recueil de J.-M. Berthelot, Sociologie. Epistémologie d'une discipline. Textes fondamentaux, Bruxelles, De Boeck, 2000 (l'objet, la théorie, les données, l'explication, la justification et la preuve, l'objectivité).

11 Nous disposons d'importantes contributions comparatives sur l'évolution de la preuve depuis l'Egypte antique aux E.-U. d'aujourd'hui, publiées dans «Recueils de la Société Jean Bodin pour l'histoire comparative des institutions », XVI, 1964, La preuve. Première partie: Antiquité; XVII, 1965, La preuve. Deuxième partie: Moyen Âge et temps modernes; XVIII, 1963, La preuve. Troisième partie: Civilisations archaïques, Asiatiques et Islamiques; XIX, 1963, Quatrième partie: Période contemporaine. Un travail exhaustif sur l'emploi effectif de la notion dans différentes civilisations et époques serait d'une grande utilité pour situer le débat à un bon niveau de généralité.

12 Aristote, Rethorica, I, 2, 1357 b 5; I, 1375a, 24 et 1368b, 7-8. Il est vrai que cet auteur distingue le syllogisme démonstratif de l'enthymème utilisé dans l'argumentation quotidienne. Le prémisses de l'enthymème sont des endoxa, des propositions admises et vraisemblables car elles paraissent évidentes à tout le monde, ou à la plupart, ou encore à des personnes compétentes. A ce propos voir G.E.R. Lloyd, Aristotelian Explorations, Cambridge, Cambridge University Press, 1996. De ce même auteur voir ce qu'il a écrit sur la conception et la pratique de la preuve en Grèce et dans le rituel védique in Demystifying Mentalities, Cambridge, Cambridge University Press, 1990, chap. III (trad. franç. Pour en finir avec les mentalités, Paris, La Découverte, 1993). Cet auteur démontre que les Grecs ont réservé une attention particulière aux critères permettant de valider ou d'invalider la preuve. Pour distinguer le discours vrai de celui qui ne l'est pas, ils ont élaboré les règles formelles qui énoncent les conditions nécessaires pour qu'une démonstration puisse être reconnue comme valide.

${ }_{13}$ Aristote, Analytica priora, II, 27, 70 b 2. Voir aussi C.Ginzburg, Aristotele, la storia, la prova, «Quaderni storici», n. 85, XXIX, n. 1, aprile 1994, pp. 51-67, à présent avec le titre Ancora su Aristotele e la storia, in Rapporti di forza. Storia, retorica, prova, Milano, Feltrinelli, 2000, pp. 51-67 (trad. franç., Rapports de force. Histoire, rhétorique, preuve, Paris, Gallimard/ Seuil, 2003, pp. 43-56). 
logique étant donné que toute démonstration implique des argumentations pareilles dans les domaines de la logique et de la rhétorique. En conséquence, la preuve se situe entre les deux.

Avec Sextus Empiricus la preuve devient le signe indicatif de l'absence de doute et le raisonnement démonstratif le producteur et le justificateur de la connaissance ${ }^{14}$.

A la suite du développement des doctrines et des pratiques médicales et juridiques, la place de la preuve selon raison (pisteusis, pistesis, action de confiance) acquiert un status plus précis ${ }^{15}$. Pour Cicéron la preuve est «argumentum», «ratio »; elle demeure dans le domaine du doute, du probable, de l'incertitude. En effet, il affirme: «Argumentum est ratio quae rei dubiae faciat fidem ${ }^{16}$. Ici fides est le calque de pistis, c'est-à-dire de la confiance en autrui, du gage de foi, de caution, de garantie, de raison de croire à quelque chose. De son côté, Quintilien élabore une théorie du probable et du normal («id quod plerumque accidit»), une théorie de la pertinence de la preuve fondée sur l'exclusion, et une théorie des centres des argumentations (status). Toutes les preuves ne sont pas pertinentes. Chaque status admet un type de preuve, étant donné qu'il «est ad quem probationes partium referantur». Les preuves techniques (pisteis entechnoi), les probationes artificielles n'appellent aucun raisonnement car elles mettent en jeu les capacités professionnelles de celui qui argumente ${ }^{17}$.

Les logiciens du Moyen Age perfectionnent et parachèvent les doctrines classiques. Mais le renouveau du débat s'opère dès le XVII siècle, notamment avec John Locke, lequel, après avoir donné de la consistance à la thèse que la preuve démontre la concordance entre deux idées et opposé la démonstration certaine à la probabilité qui ne l'est pas, théorise, enfin, la possibilité de preuves probables ${ }^{18}$. Après lui, Christian Wolff ${ }^{19}$ affirme que la preuve est un syllogisme dont les prémisses sont des définitions, des expériences indubitables formant des axiomes.

David Hume distingue tous les arguments en démonstrations, preuves et probabilités. Les preuves sont des arguments tirés de l'expérience, «dérivés de la relation de cause et effet [et] qui se trouvent complètement soustraits au doute et à l'incertitude »; les démonstrations sont établies sur la causalité, elles « dépassent la probabilité et peuvent être reçus comme une forme supérieure de témoignage »; les probabilités sont «ce qui est encore considéré avec incertitude ${ }^{20}$. En bref, il y a preuve lorsque un dispositif objectif (la méthode), ayant des effets subjectifs (la

14 Sextus Empiricus, Pirroneion hypotyposeon libri tres, II, 104, 135.

15 Pour un exposé complet de toute la question. cf. P. Butti de Lima, L'inchiesta e la prova : l' immagine storiografica, pratica giuridica e retorica nella Grecia classica, Torino, Einaudi, 1996 et A. Giuliani, Il concetto di prova. Contributo alla logica giuridica, Milano, Giuffré, 1971.

16 Cicero, Topica, II, 8.

17 Quintilianus, Institutiones oratoriae, V, 10,8.

18 J. Locke, An Essay concerning human Understanding, IV, 2, 3 et IV, 15, 1.

19 Ch. von Wolff, Philosophia rationalis sive logica, methodo scientifica pertractata et ad usum scientiarum atque vitae aptata, 1728, § 498. Pour en savoir davantage sur ce philosophe allemand, 1679-1754, lire les articles parus dans un numéro spécial des «Archives de philosophie», Tome 65, Cahier 1, janviers-mars 2002.

${ }^{20}$ D. Hume, Inquiry concerning human Understanding, 1748, VI, note; A Treatise of Human Nature, I, iii, xi. 
croyance) et intersubjectifs (la reconnaissance générale), écarte les doutes et les incertitudes et ne laisse aucune place à l'opposition.

A son tour, Immanuel Kant caractérise quatre espèces de preuves: la preuve qui va du général au particulier (c'est la démonstration), le raisonnement par analogie, l'opinion vraisemblable et l'hypothèse (recours à un principe explicatif simplement possible). Les preuves démonstratives et apodictiques se trouvent exclusivement dans le domaine des mathématiques où les concepts sont construits et «les conclusions peuvent y être immédiatement établies par intuition pure». A côté des jugements assertoriques de la foi et des jugements apodictiques du savoir, il distingue aussi les preuves empiriques des preuves philosophiques (qui sont discursives) et des preuves transcendantales (ostensive, alliant à «la fois la conviction de la vérité et l'intelligence des sources de cette vérité»; apagogique, «peut bien produire la certitude, mais non la compréhension de la vérité considérée au point de vue de l'enchaînement qui la relie aux fondement de sa possibilité»). L'incontestabilité est garantie par le recouvrement du subjectif (la certitude) par l'objectif (la pleine intelligibilité de la situation). Il y a une pertinence des preuves par analogie car elles agissent sur la conviction. Cependant Kant partage le point de vue de Hume selon lequel les preuves empiriques ne peuvent pas donner des preuves apodictiques ${ }^{21}$.

Jeremy Bentham affirme que la preuve certifie la correspondance entre les faits observés et permet de tirer des conclusions. Pour John Stuart Mill la preuve est une proposition admise en vertu d'une inférence. Elle rentre dans le domaine de la logique en tant qu'evidence. John Dewey contestera cette logique basée sur des notions méconnaissant les fonctions des concepts dans l'observation et dans la vérification des généralisations. Il fera de la vérité l'équivalent de l'utile, du pratique et de l'efficace et il distinguera la démonstration rationnelle de la démonstration ostensible, la preuve logique, déductive, nécessaire, de la preuve du sens commun fondée sur une conviction ${ }^{22}$.

La clarification de tous ces éléments est l'œuvre des logiciens et des mathématiciens contemporains. Ce sont eux à donner les contributions les plus remarquables à la construction du concept de preuve tel qu'il est véhiculé désormais par les sciences contemporaines.

De D. Hilbert à A. Church et à K. Gödel, de W. Quine à E. Nagel, la théorie de la preuve trouve son ancrage dans le principe de non contradiction. La validité est alors la correspondance entre les énoncés hypothético-déductifs et les énoncés descriptifs issus des observations contrôlées et calibrées. Certes, elle est relative car la nature de sa cohérence dépend de la cohérence du système à partir duquel elle a été élaborée. Ludwig Wittgenstein associe la problématique de la preuve à celle de la détermination du sens des propositions. La subordination du sens

I. Kant, Kritik der reinen Vernuft, $2^{\mathrm{c}}$ éd., 1787, sur la doctrine de la méthode, chap. I, section II.

22 J. Dewey, Logic.The Theory of Inquiry, New York, Allen und Unwin, 1939, ch. XII. Ce logicien fait une distinction entre les lois générales qui sont existentielles et les lois universelles qui ne le sont jamais. Cela permet de s'interroger sur ce que signifie un phénomène et d'accepter qu'il y a des compréhensions de natures différentes de ce même phénomène. Le même dans Art as Experience, New York, Putnam, 1958 (trad. italienne, Firenze, La Nuova Italia, 1973), affirme pourtant que le chercheur en sciences humaines doit avoir la créativité de l'artiste, son imagination productrice et son intuition empathique. 
cognitif au principe du contrôle empirique, lui fera écrire, dans le Tractatus logico-philosophicus, 6.1262: «La preuve en logique est seulement un expédient mécanique pour reconnaître plus aisément la tautologie lorsqu'elle est compliquée».

La preuve démonstrative est impersonnelle, ne dépend pas de l'adhésion de tel ou tel autre, elle ne peut pas prouver à la fois le pour et le contre. L'incohérence et l'incomplétude laissent les questions insolubles, c'est-à-dire des énoncés dont on ne peut prouver ni la vérité ni la fausseté ${ }^{23}$. Pour cette raison il faut faire une distinction nette entre la validité et la vérité. La première est la résultante du respect de certaines règles systématiques tandis que la seconde relève de la crédibilité.

Une systématisation de toutes ces diversités, voire leur harmonisation et recomposition en unité, est entreprise par le Cercle de Vienne (R. Carnap, H. Hahn, O. Neurath et les autres). Le néo-positivisme, en quête d'une théorie de la vérité, va donner de l'importance aux rapports vérification/sens, aux propositions protocolaires, à la neutralité du langage d'observation. Le liant de tous les travaux est fourni par le couple philosophique vrai/faux et par le postulat de l'existence d'une réalité indépendante et extérieure à celle construite par les chercheurs.

A partir des années ' 60 des critiques virulentes vont contester ces approches au profit d'une sorte de conventionnalisme, d'une approche consensuelle. La preuve n'est qu'une construction langagière, qu'une convention sociale, une croyance, le résultat d'alliances, de réseaux, de victoires dans des compétitions. La validité est réduite à la crédibilité. Thomas S. Kuhn, David Bloor, John Arundel Barnes, Karin Knorr-Cetina, Bruno Latour et bien d'autres, échafaudent une multitude d'énoncés théoriques autour de ces thématiques et alimentent des débats interminables sur les représentations de la «réalité», sur la réduction de la validité à la crédibilité, sur le rôle des communautés scientifiques dans la production et la reproduction de la preuve scientifique ${ }^{24}$.

L'analyse rapide du status réel de la preuve et des modalités pratiques des raisonnements ainsi que le travail concret de terrain suggèrent que ces débats épistémologiques n'ont exercé aucune influence sur les praticiens des enquêtes. Ni les structures logiques utilisées, ni les procédures argumentatives, ni les conceptualisations et les implications de ce «prouver par preuves», plus ou moins dégagées des contrôles formels ou empiriques, n’ont été affectés par les travaux des épistémologues et des logiciens sur la notion de preuve.

${ }^{23}$ D'autres informations se trouvent in E. Desmons, La preuve des faits dans la philosophie moderne, «Droits», n. 23, 1996, pp. 13-20; X. Lagarde, Vérité et légitimité dans le droit de la preuve, Ibid., pp. 31-39; P. Bouretz, La preuve, rationalisation et désenchantement: Autour de Max Weber, Ibid., pp. 99-106.

${ }^{24}$ G. Busino, Questions actuelles de sociologie de la science, Lausanne, Iasul, 1995; Id., La sociologie de la connaissance scientifique, Lausanne, Iasul, 1997; Id., Sociologies des sciences et des techniques, Paris, Puf, 1998; Id., Intorno alle discussioni e ricerche recenti sulla sociologia delle scienze, «Revue européenne des sciences sociales », XXXIX, 2001, n. 120, pp. 145-190. 


\section{LA MÉDECINE}

Selon une doctrine désormais canonique, mise au point avec un talent inimitable et une érudition considérable par l'historien italien Arnaldo Momigliano, l'investigation médicale et les controverses judiciaires sont à l'origine du status de la preuve ${ }^{25}$. L'examen clinique et le procès, l'un et l'autre au centre des situations individuelles, ont fixé les règles et les procédures ayant structuré, par paliers successifs, la preuve.

Contrairement à Momigliano, je crois que l'historique sur les origines du status de la preuve dans les pratiques des médecins et des juristes d'une époque lointaine, ne peut pas nous éclairer au sujet des questions de la preuve médicale et de la preuve juridique d'aujourd'hui.

A présent, une partie importante de la médecine, de la sémiotique à l'épidémiologie, relève de la culture scientifique bien qu'une autre partie dépende étroitement des aptitudes personnelles, de l'art et de l'expérience du clinicien. La preuve médicale est fondée sur l'aperception des symptômes, sur l'interprétation d'une série d'indices et sur des notions confuses, telles que les probabilités conditionnelles, les pourcentages et les fréquences naturelles ${ }^{26}$. Elle est structurée par des raisonnements plausibles, fondée sur l'induction, sur la logique de l'incertitude, de l'opinion et sur des protocoles en usage dans la corporation médicale ${ }^{27}$. Elle procède par tâtonnement dans le but d'effectuer des choix thérapeutiques cohérents et efficaces. Mais elle est aussi une croyance selon laquelle, étant donné un certain faisceau d'informations, un certain événement et un certain état pourraient advenir probables ou possibles. Une telle croyance génère, en outre, un degré de confiance, d'espoir, de crainte que quelque chose arrivera, se vérifiera ou ne se vérifiera pas. Il s'agit d'une «thèse subjectiviste» tenant compte des faits et d'informations nouvelles, le cas échéant aidant à abandonner ou à redimensionner les hypothèses initiales, à assigner une potentialité plus grande à d'autres hypothèses jugées meilleures sur la base d'autres constats, d'autres expériences et des résultats non probants des essais déjà exécutés. S'agit-il au moins de preuves probables fondées sur des inductions énumératives, dont le principe di pertinence permettrait de sélectionner ce qui mérite d'être retenu?

Les chercheurs affichent une grande retenue à ce propos. Ils parlent plutôt de principe de raison suffisante, ou principe d'indifférence dans la formulation de Laplace. Récemment on a mis en place une procédure, l' «Evidence based Medecine $\left(_{\mathrm{EBM}}\right)^{28}$, dont le modèle de référence reste la physique. La procédure est

25 A. Momigliano, History between Medicine and Rhetoric, in «Annali della Scuola Normale di Pisa », s. III, 15, 1985, pp. 767-780, et à présent in Ottavo contributo alla storia degli studi classici e del mondo antico, Roma, Edizioni di storia e letteratura, 1987, pp. 12-25.

${ }_{26}$ Su la question voir G. Gigerenzer, Reckoning with Risk. Learning to Live with Uncertainty, London, Penguin, 2000 et J.-P. Papart \& Ph. Castonay, Le raisonnement scientifique appliqué au domaine de la santé, Lausanne, Réalités sociales, 2002.

27 A. Wald, Sequential Analysis, New York, Dover, 1973 et D. R. Lindley, Making decisions, New York, Wiles, 1985.

28 Pour les anglo-saxons evidence se réfère aux moyens grâce auxquels les faits objets d'examen sont rendus évidents et prouvés. L'evidence est à la base de le proof qui porte à conclure sur la vérité ou non des faits objets de l'enquête. 
basée sur les fréquences de la théorie probabiliste, sur des hypothèses qui tiennent compte du rapport coûts/bénéfices pour le patient, de l'efficience et de la productivité des systèmes sanitaires. Il ne s'agit pas d'une théorie de la preuve, mais plutôt d'un processus logique pour arriver à prendre une décision acceptable. Locke aurait observé peut-être que la décision n'est pas prise à la lumière du jour mais au crépuscule des probabilités.

Lorsqu'on étudie les applications de cette procédure, on s'aperçoit que la preuve et ensuite l'explication ne sont rien d'autre que des corrélations statistiques plus ou moins significatives. Or la fréquence observée, celle qui permet de quantifier en termes probabilistes la force de l'association entre un effet et une cause, n'est pas seulement un numéro mais aussi une entité théorique qui explique cette même fréquence. Les corrélations n'ont aucune base empirique, elles sont des artefacts continuellement refaits moyennant des constructions et des démolitions théoriques. Elles sont des abstractions indispensables pour argumenter intersubjectivement, mais elles n'offrent aucune preuve ni en soi ni pour soi.

En fait, la preuve «EBM» est fondée sur la fréquence des relations détectées. Cette approche cache des défis insurmontables. Dire qu'un tiers de la population $X$ souffre de malnutrition ce n'est pas la même chose que de constater que dans cette population il y a des sujets avec certaines caractéristiques, celles d'homme/femme, de vieux/jeune, de citadin/campagnard, d'intellectuel/manuel, etc., affectés ou non par la sous-alimentation. En théorie des fréquences, les deux situations, pourtant différentes, ont une description identique. Si les informations contextuelles sont importantes, comment évaluer les a priori théoriques dans la détermination, par le chercheur, des éléments contextuels pour la construction de l'évidence? Sur quelles circonstances faut-il s'appuyer pour affirmer qu'une donnée est vraie ou valide? Certains disent: les circonstances disponibles pour soutenir ou non la vérité d'une proposition sont exprimées par le nombre de faits $m$ où l'événement s'est vérifié et par le nombre de fois $n$ où ils auraient pu se vérifier. Cependant cette fréquence relative néglige l'ordre dans lequel se sont déroulés les événements observés; elle délaisse les circonstances au cours desquelles les observations ont été faites. Certes, il y a des situations où l'ordre de déroulement et d'enchaînement est insignifiant, mais il y en a d'autres où il est d'une grande importance. Par exemple: j'interroge 100 patients ayant une maladie allergique et traités avec des antihistaminiques ou de la cortisone. 63\% ont été soulagés par le traitement. Il y a $63 \%$ de succès, 63 sur 100. La probabilité pour un patient d'être débarrassé de la maladie est de $0,63 \%$. En regardant de plus près, je m'aperçois que l'échantillon est composé de 50 hommes et de 50 femmes. Parmi les hommes 15 ont guéri, c'est-à-dire le $30 \%$, alors que chez les femmes 48 , c'est-à-dire le $96 \%$.

La fréquence relative se rapporte à l'échantillon total, elle néglige l'ordre, elle résume les observations «expérimentales», elle ne constitue pas un «evidence», un «proof». La fréquence ne constitue jamais la globalité de l'évidence car elle fait l'économie du contexte et de l'ordre dans lesquels les observations ont été faites.

Des remarques analogues peuvent être faite à propos de l'analyse factorielle. Utilisée lorsqu'on a un certain nombre de tests ayant entre eux des corrélations déterminées, cette analyse se propose de trouver un certain nombre de facteurs indépendants n'ayant entre eux aucune corrélation et néanmoins susceptibles de 
rendre compte intégralement de toutes les corrélations. On postule qu'ils en rendent compte lorsque, pour chaque couple de tests, la somme des produits de la corrélation du premier test avec chaque facteur reproduit la corrélation effective de deux tests. Il s'agit d'une méthode incertaine puisque: les coefficients de corrélations n'expriment pas les interconnexions des tests; le calcul des saturations (le poids d'un test dans un facteur) et des estimations des facteurs reste très problématique; la dénomination des facteurs est instable.

Dans la médecine ainsi que dans toutes les sciences humaines l'ensemble des faits et des informations disponibles qui permettent la détermination des probabilités d'une proposition, cet ensemble est réduit le plus souvent à une fréquence détachée des contextes où elle est déterminée, à des tests abstrus et c'est bien cela que dans ces sciences est dénommé «la preuve $»^{29}$.

\section{LE DROIT}

Les juristes ont réservé une attention particulière aux études sur la preuve (probationes inartificiales) $^{30}$, à la logique des propositions normatives, à la logique du raisonnement juridique, à l'ars opponendi et respondendi, aux vérités formelles $^{31}$. Dans les disciplines juridiques la distinction entre la théorie et la pratique est difficile à établir étant donné que la recherche de la vérité n'est pas le but du droit de la preuve. En effet, la preuve doit servir à vérifier un fait particulier, à le contrôler, à le légitimer, à tenir à distance les incertitudes et les perplexités. Pour cette raison la chaîne du raisonnement juridique doit éliminer toutes les contradictions, les ambiguïtés, les incertitudes, les «vide», moyennant quoi elle débouche inévitablement sur une solution devant être à la fois nécessaire et incontestable.

La preuve en droit est fondée sur une rationalité qui lui est propre, différente de la rationalité scientifique. Elle est l'expression d'une ou de diverses rationalisations. Elle a deux faces: 1 . celle d'une logique déductive que l'on peut inférer d'un ordre clos et complet et que l'on peut interpréter de façon sémantique et structurale; 2 . celle ayant pour objet des faits qu'on analyse en termes pragmatiques, c'est-à-dire de façon sémantique et fonctionnelle. Le logicisme déductif dérivé de la loi abstraite, attentif à l'évaluation et au contexte, s'accommode, il est

Même dans la conception poppérienne des sciences sociales l'importance de la notion d'analyse situationnelle est rappelée avec insistance. Voir la présentation qu'en a faite A. Boyer, Introduction à la lecture de Karl Popper, Paris, Presses de l'ENS, 1994, pp. 191-206.

3o Cf. P. Béliveau \& M. Vauclair, Traité général de preuve et de procédure pénales, Montréal, Thémis, 1999; F. Carnelutti, La prova civile. Parte generale. Il concetto giuridico della prova, Milano, Giuffré, 1992; P. De Lalla, Logica delle prove penali. Introduzione, archeologia, semantica normativa, logica inquisitoria, Napoli, Jovene, 1973; V. Denti, Processo civile e giustizia sociale, Milano, Comunità, 1971; L. Ducharme, Administration de la preuve, Montréal, Wilson \& Lafleur, 2001; X. Lagarde, Réflexion critique sur le droit de la preuve. Préface de J. Ghestin, Paris, LGDJ, 1994; P. Pactet, Essai d' une théorie de la preuve devant la juridiction administrative, Paris, Pedone, 1952; C. Pernel, Libertés et droits fondamentaux dans la recherche de la preuve pénale en médecine légale, Villeneuve d'Ascq, Septentrion, 2000.

31 S. Humphreys, Law as Discourse, «History and Anthropology », I, 1984-1985, pp. 241-264, avec une importante bibliographie. 
compatible avec le logicisme inductif, soucieux des faits et des définitions. Les invariants (la preuve, l'évaluation) et les variables contextuelles (moyens de preuve, éléments de preuve, vérifications) prévalent sur les contenus et les catégories subjectives (évidence, apparence, certitude, incertitude, doute, crédibilité, indice, soupçon). Il y a, dans un même processus herméneutique deux catégories de règles, celles juridiques d'interprétation et les logiques d'inférence. Les preuves parfaites sont les actes instrumentaires, l'aveu et le serment. Les procédures de vérifications sont limitées par leur recevabilité. Le juge statue même sur les fondements de preuves imparfaites pour autant que certaines règles aient été respectées. Il exerce un pouvoir en décidant que tel fait particulier, ou tel acte individuel précis, a les caractéristiques prescrites pour l'application de la règle générale. Pour l'exercice de ce pouvoir le juge scotomise tous les éléments du contexte qui ne se rapportent pas, d'une façon ou d'une autre, à l'activité de l'auteur du fait litigieux, au problème qu'il doit résoudre. Pour cette raison il décide que tel récit est conforme à la vérité ou plus proche d'elle, que tel autre n'est guère pertinent ou recevable. Le verdict impose une décision, contraignante pour les parties. Celle-ci est définitive, sauf fait nouveau estimé tel par une instance supérieure. Il rend sa décision sur la base de normes légales et en se référant à des principes généraux, substantifs et procéduraux.

La décision, la sentence, a la forme d'un syllogisme dont la majeure est l'énoncé abstrait de la loi, la mineure est un fait concret et la conclusion est l'inférence de la décision.

La nature de la preuve juridique est très différente de la preuve historique ou de la preuve expérimentale. Entre la vérité scientifique, la vérité historique et la vérité judiciaire existe une incompatibilité radicale. Le juge doit résoudre une quaestio facti, qui lui a été soumise par les parties (nemo iudex sine auctore), d'après une quaestio juris. Son activité, gouvernée par les lois positives, se borne à l'examen des faits qui lui ont été soumis (iudex secundum allegata decidere debet; quod non est in actis non est in mundo). L'historien, par contre, choisit son sujet, formule ses questions et utilise tous les matériaux qu'il juge utile pour y répondre. Les caractéristiques de la preuve judiciaire ont pour fonction de faire naître une conviction sociale alors que la preuve scientifique vise à établir une vérité objective bien que provisoire, indifférente à l'utilité sociale. Les propositions découlant d'une présomption sont jugées comme descriptives du cours normal des choses tandis que l'imputation d'un risque devient une méthode pour résoudre des litiges (un exemple nous est donné par le principe de précaution). La preuve juridique vise à légitimer les décisions de l'autorité et des institutions, à structurer des représentations comme celles d'adhésion, de faute ou encore de négligence. La décision judiciaire s'impose inter partes; elle est la solution donnée à la controverse. Ce n'est jamais le cas en historiographie. Alors que le juge doit donner la solution aux questions que lui ont été posées, l'historien peut suspendre son jugement et laisser à d'autres historiens la charge des questionnements et la quête d'autres archives ${ }^{32}$.

Toute cette problématique a été au centre d'un important débat, en Italie, à l'occasion de la publication d'un livre du philosophe Guido Calogero, La logica del giudizio e il suo controllo in Cassazione, Padova, Cedam, 1937. Benedetto Croce en a discuté les thèses in «La Critica», XXXV, 1937, pp. 378-379 et puis in Conversazioni critiche, Bari, Laterza, 1939, V, pp. 279-283. Les inter- 


\section{LA PRÉHISTOIRE ET L’ARCHÉOLOGIE}

Dans les disciplines de la Préhistoire (la Paléontologie, la Paléoanthropologie, l'Archéologie préhistorique et la Paléoethnologie) le rassemblement des éléments d'une preuve est très limité. Les documents, les témoignages, souvent incomplet, incertains, douteux, n'apportent que des informations limitées et les pièces comparatives sont disparates, parfois même inexistantes. Ces disciplines pratiquent le raisonnement par eikos, régressif des conséquences aux principes. L'interprétation des données disponibles est fondée sur ce qui n'est pas donné. Cette forme de connaissance est purement conjecturale. Elle ne permet pas de pondérer les témoignages et de donner une bonne évaluation critique des informations.

La connaissance indirecte par les traces permet quelquefois de remonter à l'événement par un raisonnement des effets aux causes. Il n'en reste pas moins que ce prétendu raisonnement causal comporte des suppositions, des alternatives de causes possibles, des lacunes attribuées au hasard. Les critères pour déterminer la pertinence des informations retenues et des langages décrivant la logique des opérations effectuées, restent indéterminables selon des procédures répétables ${ }^{33}$.

L'archéométrie, la stratigraphie, les repérages en plan et en niveau, et les autres méthodes physiques devraient permettre l'établissement de séquences spatiales et temporelles sûres, des relations réciproques entre les unités stratigraphiques, néanmoins elles suscitent doutes et perplexités nombreux sur le lointain passé des premiers hommes. L'application de nos savoirs actuels à des sociétés disparues à jamais et sur lesquelles nous n'avons que des données incertaines ou impressionnistes, rend fallacieux le raisonnement causal. Le recoupement des traces et la convergence des indices n'arrivent pas à effacer les suppositions, à combler les lacunes, à hiérarchiser les causes alternatives ou possibles, enfin à bâtir des preuves.

Les chercheurs transforment une information imprécise en preuve d'où ils soutirent une hypothèse transformée aussitôt en un fait établi. Ensuite ils utilisent, selon J.-M. Pailler, «la plateforme hypothétique comme si les documents qui la constituent étaient démontrés, affirmés. A partir de ces données déjà fragilisées, on établit d'autres hypothèses qui à leur tour servent de plate-forme à des nouvelles hypothèses ». La documentation fragmentaire, épisodique, parfois

ventions du juriste Piero Calamandrei ont été publiées in Studi di storia e diritto in onore di Enrico Besta per il XL del suo insegnamento, Milan, Giuffré, 1939 et dans la «Rivista di diritto processuale civile», I, 1939. Elles ont été analysées par Benedetto Croce in «La Critica», XXXVII, 1939, pp. 445-446, puis in Pagine Sparse, Napoli, Ricciardi, 1943, III, pp. 344-346 et in Pagine Sparse, Bari, Laterza, 1960, III, pp. 447-450. Une version définitive du texte de P. Calamandrei, Il giudice e lo storico se trouve dans Studi sul processo civile. Vol.V: Teoria generale e metodo, Questioni di dottrina e di giurisprudenza, Legislazione comparata (1938-1943), Padova, Cedam, 1947, pp. 27-51.

33 Malgré les affirmations de J.-Cl. Gardin, Une archéologie théorique, Paris, Hachette, 1979, d'après lequel: «Les inférences que nous pratiquons, à partir de matériaux recueillis sur le terrain, sont constamment exposées à des épreuves de vérification (provisoire, s'entend, mais comme on l'entend aussi dans les sciences naturelles) à la suite desquelles une partie d'entre elles viennent grossir ou modifier le savoir 'établis' dans la 'communauté savante'...». De cet auteur voir aussi Entre modèle et récit: les flottements de la troisième voie, in Le modèle et le récit, sous la direction de J.-Y. Grenier, C. Grignon, P.-M. Menger, Paris Ed. de la MSH, 2001, pp. 457-488. 
unique, n'oblige pas à recourir, pour fixer la validité, au principe testis unus, testis nullus. Les lacunes sont comblées au moyen d'extrapolations et de généralisations. Avec le stratagème bien connu en dialectique éristique, d'un fait notoire certifié dans un contexte on tire une induction que l'on fait valoir ailleurs ${ }^{34}$.

Ainsi on peut passer de l'homme de Neandertal à l'homo sapiens Idàltu, de la théorie que l'homo sapiens était apparu en plusieurs endroits du monde au même moment à une autre affirmant qu'il a son origine en Afrique. La preuve serait donnée par des subtiles variations morphologiques (longueur et robustesse) des restes d'un crâne déterré dans la région d'Afar, en Ethiopie, et que les isotopes d'argon datent de 150.000/160.000 ans. Les fortes imprécisions à propos de la datation, la mauvaise qualité des restes déterrés, l'impossibilité de les comparer avec d'autres matériaux proches, ne semblent pas trop perturber la certitude des chercheurs.

L'exemple de la disparition de la civilisation Mayas paraît paradigmatique. Le déclin aurait été déterminé par des perturbations climatiques, par les sécheresses qui auraient épuisées les eaux des réservoirs souterrains des cités et déstabilisé le pouvoir politique chargé des approvisionnements. Dès lors les Mayas auraient abandonné, en plusieurs phases, notamment dans les années 810, 860 et 910 de notre ère, leurs centres religieux du Sud. La preuve de cette affirmation serait donnée par la reconstitution, au moyen d'analyses géologiques et chimiques, des régimes des pluies et de l'intensité des précipitations, en bref par le rôle de l'eau dans la survie des hommes et des sociétés.

L'accord entre les chercheurs est utilisé aussi comme étant une preuve. Pour cette raison le recours régulier aux prejudicia (thèses identiques ou analogues acceptées par des savants reconnus), aux rumores atque fama («on retient communément», «il est désormais assuré», «il est bien établi», etc.) est une pratique courante dans ce genre de recherches.

Les prémisses de l'enthymème sont placées sur deux types de signes: le semeion et le tekmerion. Les deux instaurent une implication: si $a$ existe alors $b$ existe aussi; si $q$ alors $p$. Une tache noire dans un terrain peut indiquer la pourriture d'un poteau qui était là, mais aussi bien d'autres choses que nous ignorons et que nous ne savons pas prendre en considération.

Parfois on construit un modèle, au moyen des données générales sur les sociétés humaines connues et réputées proches de celle qu'on est en train d'étudier, modèle qu'on essaie puis de vérifier avec les archives archéologiques disponibles. Il a été écrit: «De toute façon, quelle que soit la méthode, on finit toujours par projeter sur le document découvert et sur sa place dans l'organisation spatiale l'image de ce que l'on connaît. Le préhistorien ne cesse de concevoir la vie des hommes préhistoriques comme étant proche de celle des sociétés primitives et maintenant, pour les périodes les plus anciennes, proche de celle des grands singes anthropoïdes.» $^{35}$

34 A. Schopenhauer, Eristische Dialektik oder die Kunst Recht zu behalten, 1831, à présent in Sämtliche Werke. Herausgeber A. Hübscher, Wiesbaden, Brockhaus, 1972.

35 J. Chavaillon, La preuve en Préhistoire ou les limites de la crédibilité, «Le temps des savoirs», n. 5, janvier 2003, pp. 53-73 
Dans ces disciplines on trouve, on découvre, on collecte, on analyse et on classe des données ${ }^{36}$. L'analyse stratigraphiques consiste à énoncer des prédicats, la plupart du temps binaires: la strate $a$ couvre la strate $y$, laquelle à son tour couvre la strate $z$. L'amphore trouvée a ou n'a pas les deux anses. Le lécythe a ou n'a pas l'embouchure évasée. Tout cela permet d'organiser la réalité connue selon des critères cohérents de nature inductive, mais tout cela n'explique pas la qualité des objets ni les causes des événements.

Formuler une hypothèse reliant les données entre elles afin de produire une représentation cohérente, c'est une activité intellectuelle que Charles Sanders Peirce a appelé l'abduction. Il s'agit d'un type d'inférence (le signe, l'objet, l'interprétant) qui fournit une hypothèse explicative d'un ensemble de faits. La prémisse majeure du syllogisme est certaine, mais la mineure est probable, donc l'inférence peut ne pas être vraie. Le lécythe est un vase funéraire grec, il provient donc d'un tombeau grec. Les contextes similaires, les comparaisons typologiques, ethnographiques, anthropologiques, la tradition, les récits mythiques ne consentent pas d'être certains au sujet de la provenance. Dès lors, est-il légitime de déduire des conséquences en fonction d'une telle hypothèse? Vérifier cette hypothèse à la lumière d'autres données? Cette induction permet d'appliquer l'explication à tous les autres cas? Il n'est même pas sûr que l'on puisse s'appuyer sur le «Covering-Law Model» dans la formulation de Carl Hempel.

Le modus operandi de ces disciplines est régressif: partir d'un indice pour arriver à des présupposés. Cela est problématique puisque le chercheur, lors d'une analyse stratigraphique, doit donner du sens aux données moyennant conjectures, des prophéties rétrospectives, des abductions non codifiées, non basées sur un savoir typologique mais sur des symptômes et sur des indices ambigus. En établissant des analogies entre des observations faites dans des contextes différents mais jugés ou réputés apparentés à un titre quelconque, le chercheur doit jouer sur des relations entre les objets d'une part et entre les contextes de l'autre afin d'avoir des typologies génératrices d'assertions. Le statut de ces dernières reste hypothétique. Ces assertions ne constituent pas une preuve ni validée ni validable ${ }^{37}$.

\section{LA GÉOGRAPHIE ET LA DÉMOGRAPHIE}

Le cas de la géographie est, à plus d'un titre, exemplaire. Cette discipline a emprunté sa systématique de la preuve tout d'abord à la science politique, puis aux sciences naturelles (géologie, pétrographie, minéralogie, climatologie, etc.),

36 Voir à ce propos J.-M. Pailler, Archéologie qui trouve, archéologie qui prouve?, «Le temps des savoirs », n. 5, janvier 2003, pp. 125-161. Le livre récent de Michel Reddé, Alésia. L'archéologie face à l' imaginaire, Paris, Ed. Errance, 2003, fait l'histoire des recherches pour la localisation de l'endroit où César remporta la victoire sur Vercingétorix. Nonobstant qu'il y ait une discordances entre le texte de César et les découvertes archéologiques on prétend que cette localité se situe autour de Alise-Sainte-Reine (Côte d'Or) alors que des indices concordants et des nombreuses pièces trouvées autour du Mont Auxois (les armes et une inscription) feraient plutôt pencher la balance du côté de la localité du Doubs. C'est dire combien, dans ce domaine, il est difficile à trancher la querelle au moyen d'une preuve incontestable.

37 A. Gallay, L'archéologie demain, Paris, Belfond, 1986. 
ensuite à l'histoire, puis à la mécanique sociale, à l'économie, à la statistique et aux méthodes quantitatives ${ }^{38}$, et même à la psychologie (perception de l'espace et de l'environnement). Il lui est arrivé de faire des emprunts aussi aux programmes gestionnaires et technocratiques et de montrer de l'enthousiasme pour l'écologie et l'aménagement du territoire. Rappelons pour mémoire que la preuve de la théorie spatiale a été assurée par des modèles économiques, tandis que la preuve des trames concentriques, des trames radiales et des noyaux juxtaposés fait appel aux théories sociologiques de la stratification sociale.

Entre 1970 et 1980 cette discipline a connu des bouleversements exceptionnels. On est arrivé à qualifier les débats théoriques et les discussions sur des questions abstraites de «révolution scientifique». Celle-ci paraissait annoncer un «nouveau paradigme», liquidateur de la «science normale». Cependant, «Révolution après révolution, paradigme après paradigme, et aussi fructueux qu'aient été les débats, ils n'ont pas débouché sur des résultats consensuels. La remise en cause du dogme de la géographie classique se traduisit de manière presque immédiate par l'éclatement de la discipline en différents courants.» Depuis, malgré l'avis contraire de la critique postmoderne laquelle a lié la prégnance des faits économique à la «fin de l'histoire», la discipline semble être sortie du marasme intellectuel. J. Lévy a parlé d'un «tournant géographique» et selon Staszk l'ouverture actuelle à la sociologie et à l'économie, le recours régulier à des nouvelles techniques, la «conjoncture favorable, la diversité des approches en géographie apparaît davantage comme une force que comme une faiblesse $»^{39}$.

Le cas de la démographie est encore plus frappant ${ }^{40}$. Elle affiche une confiance absolue dans l'évidence des déductions mathématiques et/ou dans l'observation statistique des données. La preuve de la baisse de la fécondité observée dans les années '70 est prouvée, par exemple, par les mesures des flux et des stocks (décès, naissances, etc.) des populations et par le postulat que les femmes ont ou non une conception séquentielle de la fécondité. Les conduites en matière de procréation et de contraception n'étant pas inscrites dans la logique d'une structure de parenté et de la prise en charge élargie des enfants, on va chercher les preuves ailleurs. Et on les trouve alors dans la diffusion de la contraception, ou dans le travail professionnel de la femme, ou dans la législation égalitaire entre les sexe, ou dans les divorces più faciles, ou dans les nouveaux modèles familiaux, ou dans la partage des tâches, ou dans les contraintes sociales ou, enfin, dans les transformations radicales induites par la globalisation des styles de vie. Malgré les indices de fécondité et de mortalité, malgré les comparaisons, malgré les raisonnements sur

${ }_{38}$ A ce propos lire l'étude précise et minutieuse de D. Pumain et M.-C. Robic, Le rôle des mathématiques dans une "révolution» théorique et quantitative: la géographie française depuis les années 1970, «Revue d'histoire des sciences humaines», n. 6, avril 2002, pp. 123-144.

39 J. Lévy, Egogéographie. Matériaux pour une biographie cognitive, Paris, L'Harmattan, 1995; Id., Le tournant géographique. Penser l'espace pour lire le monde, Paris, Belin, 1997; J.-F. Staszak, La géographie, in Epistémologie des sciences sociales, op. cit., pp. 77-116.

${ }^{40}$ H. Le Bras, dans Naissance de la mortalité. L'origine politique de la statistique et de la démographie, Paris, Gallimard/Le Seuil, 2000, s'interroge sur le statut de cette discipline (science naturelle ou bien doctrine politique?). Du même cf. Logique et autorité dans l'argumentation en démographie: une expérience, «Revue européenne des sciences sociales », XXXV, 1997, n. 107, pp. 159168. 
les similitudes et sur les différences entre des facteurs exogènes, les preuves apportées manquent de consistance, elles ne disent rien sur pourquoi la fécondité ici s'effondre et là remonte. Le recours aux déterminants économiques, ou biens à d'autres déterminations, ne fait que renvoyer au prouver d'autres comportements, eux-mêmes non prouvés.

Il en va de même pour l'augmentation des femmes dans la population active. S'il s'agit d'une augmentation de la proportion des femmes dans cette population, comment expliquer ce changement par rapport à la situation des années '50? Fautil l'expliquer par la structure par âge de la population active, par des meilleures perspectives professionnelles conquises par les femmes, ou bien par une modification de nos attentes sociales? Ou encore par les répercussions sur le niveau de vie des femmes et de leurs familles?

Il faut bien reconnaître que ces disciplines ne disposent d'aucun critère absolu d'évaluation des théories. Ces sciences réduisent la rationalité au principe de raison suffisante dont la causalité n'est qu'un des aspects et l'explication une implication dans le fait. Rares sont les démonstrations en tant que suites finies de propositions déduites par la règle de déduction à partir d'axiomes et de propositions déjà démontrés. Les raisonnements conjecturaux (l'induction et l'abduction), les logiques non standard (intuitionnistes et modales, monotones et floues) n'ont pas facilité jusqu'ici l'élaboration des déductions, même atypiques, ni permis de distinguer le vraie du faux, la vérité d'une proposition de la vérité d'une modalité.

Comme toutes les autres sciences humaines, ces disciplines utilisent la langue naturelle, ambiguë en soi; elles ne sont pas soumises à des règles formelles, à des développements contraignants; elles se servent d'axiomes pris dans des corpus mal définis, approximatifs, la plupart du temps implicites. Même les modèles, fondés sur des hypothèses descriptives et sur des mesures sans théories, ne produisent pas des implications dérivables des prémisses primitives. L'irréalisme des hypothèses initiales favorise, en conséquence, les interprétations normatives des données au détriment de celles descriptives ${ }^{41}$.

Tous les chercheurs savent que les conduites individuelles et les actions collectives, les représentations sont variables, indéterminées, adaptatrices, peu uniformes et diversement standardisées. Cependant ils continuent à utiliser des théories présupposant que la régularité des conduites est déterminée par une structure et des fonctions sous-jacentes. Dans un situé au-dessous se trouveraient réunis des éléments hétéroclites (la tradition, les normes, les règles, les habitudes, les dispositions, les routines, les capacités, l'habitus), des savoirs non propositionnels (connaissances pratiques, savoir-faire, savoirs tacites), des significations et des fonctions plus ou moins indescriptibles et invérifiables.

Ces présomptions rendent la problématique de la «preuve», dans les sciences sociales, par trop paradoxale, les recherches sur les pratiques réelles du prouver rares, et les accords entre les chercheurs sur la définition de la preuve génériques et précaires. Et pourtant le mot «preuve» revient, avec des acceptions hétérogènes, dans toutes les recherches, quantitatives et qualitatives. Même l'histoire du mot ne clarifie pas tant de conversions sémantiques et d'usages protéiformes. Tout

${ }^{41}$ P. Naville, Sociologie et logique. Esquisse d' une théorie des relations, Paris, Puf, 1982. 
au plus elle dégage les divergences existantes entre la théorie et la pratique, nous informe sur l'irréalisable coopération des théoriciens avec les chercheurs de terrain.

\section{L'ÉCONOMIE}

Cette discipline se veut la plus théorique et la plus formalisée des sciences humaines, la plus proche des sciences naturelles ${ }^{42}$. Elle privilégie le raisonnement hypothético-déductif, mathématique mais pas nécessairement quantitatif. Pour bâtir les preuves elle utilise l'histoire économique, les mathématiques, la statistique et la simulation. Or les matériaux historiques, par exemple sur les cycles économiques, sont inadaptés aux séquences actuelles tandis que les méthodes statistiques doivent se plier aux contraintes de la simultanéité des variables économiques. Il en dérive l'impossibilité, en économie, de répéter et de vérifier les recherches, de calculer des paramètres structurels ou de tester formellement des hypothèses. Puisque le recours explicite à l'observation empirique est impraticable, l'aune de la preuve reste la cohérence interne à partir du principe posé au départ. Pour cette raison il y a des économistes affirmant que la formation du capital n'apporte qu'une faible contribution à la croissance tandis que d'autres en font la condition du take-off. On peut faire le même constat pour la consommation (elle réagit ou ne réagit pas à un abaissement temporaire de l'impôt, aux effets d'annonces des futures politiques fiscales) ou encore pour l'instabilité financière sur l'activité économique réelle ${ }^{43}$.

L'approche par la dynamique des systèmes, la maximation sous contrainte, l'équilibre comme interdépendance et compatibilité mutuelle, les choix sans ou en situation d'incertitudes, l'idée de renverser les relations causales pour étudier la marche des économies, entraînent les économistes à préférer les modèles stables, à rejeter ceux qui ne le sont pas, à pratiquer une expérimentation artificielle ${ }^{44}$. L'économie vise moins à décrire et à expliquer qu'à prescrire des orientations et à préconiser des solutions en matière d'allocation des ressources et de leur utilisation. Elle contient des instructions eidétiques dont la normativité est indiscutable. Pour garantir sa neutralité du point de vue axiologique, les chercheurs ont mis au point la distinction fait/norme, laquelle, en vérité, n'est qu'une construction philosophico-sociologique. L'équilibre, mieux les propriétés d'équilibre des systèmes dynamiques ont été empruntées aux formalisations du mathématicien David Hilbert et à sa représentation du système au moyen d'un vecteur de l'espace.

${ }^{42}$ Voir le dossier «L'économie, entre sciences humaines et sciences de la nature» publié par la «Revue d'histoire des sciences humaines», n. 7, octobre 2002.

${ }^{43}$ Voir à ce propos l'introduction de Pierluigi Ciocca, Clio, nella teoria economica, à Le vie della storia economica, Bologna, Il Mulino, 2002, spéc. les pp. 9-49. Cette anthologie rassemble des essais de G. Viaggi, P. Sylos Labini, G. Lunghini, G. Gilibert, G. Becattini, P. Bini, N. De Vecchi, M. de Cecco, A. Roncaglia, M. Friedman, P. A. Samuelson, L. L. Pasinetti, sur les rapports entre la théorie économique et l'histoire.

44 Une défense de cette approche in A. Mattei, Micro-économie expérimentale, Genève, Droz, 2002. D'autres points de vue sont exposés in B. Walliser, La science économique, in Epistémologie des sciences sociales, op. cit., pp. 117-147. 
L'économie accorde une place déterminante aux données statistiques, considérées en tant que reflet de la réalité. On néglige le fait que cette réalité est appréhendée par un codage. Les tableaux à double entré de la comptabilité nationale sont des constructions. L'indice (chômage, prix, croissance, etc.) est un enregistrement, il est le résultat d'une procédure reflétant les structures administratives, juridiques e politiques du pays. Les théories sur le comportement des consommateurs, sur les courbes d'indifférences, sur la transitivité des préférences, sur le choix sans ou avec incertitude, sur les marchés et les effets externes, etc., ce sont des constructions. Il est pratiquement impossible de faire varier, de manière contrôlée, les paramètres des phénomènes dont ces théories prétendent rendre compte. La description des faits et la constatation des résultats n'interviennent qu'au terme d'un processus empirique et théorique, lequel produit une interrogation sur la réalité. L'observation en économie est inséparablement processus et résultat. Certes, les économistes ne sont pas eux-mêmes les producteurs des données utilisées. Parfois, ils se content d'analyser les données chiffrées et les observations construites par d'autres. Il s'agit d'un travail différent du travail de celui effectué dans les sciences empiriques. En physique, pour découvrir la loi gouvernant un phénomène, il faut une série de faits organisés méthodiquement par la variation d'éléments définis au préalable. Cela constitue une expérience ayant valeur de preuve. En économie, des suites d'observations apparentées permettent tout au plus de faire des comparaisons. Pour l'interprétation des données économiques, la connaissance intime du processus qui les a produites est indispensable.

Les vérifications en économie nécessitent en plus de la statistique, également de l'histoire. Mais l'une et l'autre mettent en place des situations artificielles, anachroniques, impossibles à transposer à des cas réels d'aujourd'hui. Ajoutons, à titre d'exemple, que les recherches sur les budgets des familles ont servi à prouver pendant longtemps que le salaire ouvrier suffisait ou non à la reproduction de la force travail, «à se reproduire». Mais dès les années '50 ces genres d'enquêtes, étendues à l'ensemble des métiers, devaient prouver que le salaire en tant que coût est moins important que la consommation en tant que composante de la demande globale. Pendant des années on a proclamé que les prix et les salaires sont parfaitement flexibles, qu'il y a égalisation entre l'offre et la demande sur les marchés, alors que actuellement on affirme avoir la preuve que le chômage et l'inflation assurent une certaine rigidité dans l'espace et dans le temps. Le PIB, outil primordial pour la macroéconomie keynésienne, depuis les années '60 sert uniquement à fixer les montants des subventions de Bruxelles, à évaluer les taux des déficits budgétaires, à comparer des performances budgétaires nationales.

L'économie dépend peu du passé. La statistique n'arrive pas à rendre compte de la mutuelle dépendance des phénomènes ni de la simultanéité des variables. L'économie est surtout déterminée par l'avenir, c'est-à-dire par les anticipations, les attentes, les aspirations que font les agents sur cet avenir, selon des mécanismes encore mal connus des évolutions vécues et de la compréhension que les dits agents en ont. Les théories des anticipations adaptatives et les anticipations rationnelles ne sont pas réalistes; elles ne sont même pas conformes aux processus cognitifs des agents tels que les neurosciences actuellement les présument. 
Pour toutes ces raisons les théories et les modèles, dans la science économique, ont une fonction prescriptive, normative ${ }^{45}$, presque jamais une fonction cognitive.

\section{LA SOCIOLOGIE}

Cette discipline a pour ambition d'interpréter l'expérience sociale des humains et de donner une cohérence à l'ensemble de ces interprétations ${ }^{46}$. Elle prétend fournir les matériaux constituant la base des représentations informées que les sociétés se forgent d'elles-mêmes et sur elles-mêmes; elle revendique le droit de fournir une interprétation serrée, ordonnée, du sens de l'expérience sociale des hommes encore que la théorie ait sa logique interne, que le monde dans lequel nous vivons change en continu, que le rapport de l'expérience et de la théorie reste abscons et que la vérification d'une théorie par l'expérience soit assez aléatoire. La connaissance n'est pas entièrement extérieure et ce dont nous parlons n'est pas réductible à des théories sur un objet ou un processus, c'est un rapport dans lequel nous nous trouvons à un moment donné. Fondée sur l'irréductibilité des faits sociaux aux faits psychologiques, la connaissance sociologique renferme des caractères assez particuliers et collecte des questions cruciales telles que celles-ci: Comment attribuer des pouvoirs causaux à des phénomènes sociaux puisqu'ils ne sont pas indépendants de nos perceptions, de nos croyances, de nos préférences, de nos actions, de nos réactions à des agents, à leurs propriétés, et des définitions que nous en donnons? Comment traiter des formations discursives produites au moyen des théories multiples à l'origine de descriptions différentes et impliquant des ontologies ayant par là même une fonction cognitive structurante dans la production et dans la reproduction de la réalité sociale? Comment attribuer la valeur de preuve à des constructions élaborées dans un contexte donné, par des agents donnés, en vue d'objectifs donnés, à des preuves qui sont, en somme, des activités sociales? Comment réduire à unité, de façon analytique, la pluralité de ces formes de connaissances élaborées et partagées socialement, et dont la visée pratique prioritaire est de formater une réalité commune à des collectifs sociaux donnés? Si celui qui connaît est en dehors de ce qu'il connaît, sans relation avec lui, ce qu'il connaît à travers les théories, n'est-ce qu'une relation à l'objet, à la nature, n'est-ce que l'investigation de l'objet ou de la nature qu'il est en train de pratiquer? Si le chercheur se rallie à des connaissances qui admettent le principe de complémentarité, alors décrit-il la «réalité» à partir de deux théories exclusives l'une de l'autre et pourtant également nécessaires pour la compréhension du phénomène étudié? Les catégories et les types idéaux permettent l'observation empirique et la collecte des statistiques destinées à valider les «faits» mais au prix d'une délimitation du champ des observations des relations.

A. Gibbard \& H.Varian, Economic Models, «Journal of Philosophy», 75, 1978, pp. 664-677; R. Sudgen, Credibles Worlds. The Status of Theoretical Models in Economics, «Journal of Economic Methodology», 7, 2000, pp. 1-31; F. Guala, The Logic of normative Falsification. Rationality and Experiments in Decision Theory, Ibid., pp. 59-63; M. Morgan, Finding a Satisfactory Model, in N. de Marchi ed., The Popperian Legacy in Economics, Cambridge, Cambridge University Press, 1988, pp. 199-211; C.W.J.Granger, Empirical Modeling in Economics. Specification and Evaluation, Cambridge, Cambridge University Press, 1999. 
Les constructions des sciences de l'homme se rapportent à des situations individuelles; elles sont empathiques car celui qui connaît s'identifie à ce qu'il connaît. Ces sciences produisent des connaissances authentiques, mais pas des connaissances vraies puisque leurs démonstrations ne possèdent pas les propriétés des énoncés scientifiques. Pour la sociologie ces contraintes sont encore plus implacables et incontournables.

Les travaux sur les pratiques des sociologues en matière de preuve sont peu nombreux ${ }^{47}$. Dans la pratique quotidienne de la sociologie, la résonance des acquis des débats épistémologiques et méthodologiques y est assez faible. En sociologie la théorie de la preuve n'a pas fait de progrès substantiel depuis les Règles de la méthode sociologique (1895) de Durkheim. Son modèle physicaliste postule que la méthode comparative et la méthode des variations concomitantes permettent l'expérimentation indirecte, garantissent la causalité et celle-ci est l'assurance de la validité de la preuve. Les preuves sont produites grâce à la recherche quantitative, à la statistique, au schéma causal. La recherche qualitative a un status inférieur car elle n'arrive pas à démontrer ses hypothèses, d'où l'évanescence de ces preuves ${ }^{48}$. Durkheim, on le sait, ne fait que reprendre les doctrines d'Henri Poincaré et d'Ernst Mach, il n'ajoute rien à ce que les physiciens disaient déjà à son époque, il ne donne aucune indication au sujet des procédures de recherche propre à la sociologie. Il répète que les faits sociaux sont des choses, qu'ils n'ont rien à voir ni à faire avec la psychologie. A aucun moment Durkheim n'arrive à élaborer une théorie véritablement autonome par rapport à l'utilitarisme économique, à l'organicisme biologique, à la psychologie de la Gestalt, à la philosophie de l'histoire du marxisme, aux modèles à son époque prépondérants en physique. Ce sont ces modèles scientistes, transformés souvent en métaphores, appliqués analogiquement, qui ont nourri la sociologie jusqu'à aujourd'hui.

L'assimilation implicite du monde social au monde physique et la foi dans le pouvoir explicatif des outils de la «reine des sciences », nous ont fait transposer mécaniquement dans les sciences sociales tout ce qui avait produit quelques résul-

46 Il y a des questions que les sociologues se posent continuellement: la sociologie doit-elle servir à quelque chose, avoir une quelconque utilité (politique, thérapeutique, cognitive)? Les réponses sont aussi nombreuses que les sociologues en activité. Voir à ce propos: J. Law (Ed.), Power, Action and Belief. A New Sociology of Knowledge, London, Routledge and Kegan Paul, 1986, et Sous la direction de B. Lahire, A quoi sert la sociologie?, Paris, La Découverte, 2002.

47 H. Lévy-Bruhl, Preuve judiciaire. Etude de sociologie juridique, Paris, Rivière, 1964, passe en revue les différents modes de preuves: archaïque (ordalie, duel judiciaire, serment, etc.), moderne (écrit, témoignage, commune renommée); J.-M. Berthelot, L’intelligence du sociale. Le pluralisme explicatif en sociologie, Paris, Puf, 1990, pp. 185-233; Id., Les vertus de l'incertitude. Le travail de l'analyse dans les sciences sociales, Paris, Puf, 1996; Id., Les sciences du social, in Epistémologie des sciences sociales, op. cit., pp. 203-265; C. Topalow, «Expériences sociologiques»: les faits et les preuves dans les thèses de Maurice Halbwachs (1909-1913), «Revue d'histoire des sciences humaines », I, 1999, pp. 11-46.

48 Une excellente présentation de la théorie durkheimienne se trouve in S. Moscovici, La machine à faire des dieux. Sociologie et psychologie, Paris, Fayard, 1988. Voir également J.-M.Berthelot, 1895. Durkheim. L'avènement de la sociologie scientifique, Toulouse, Presses Universitaires du Mirail, 1995; M. Borlandi et L. Muchielli (dir.), La sociologie et sa méthode. Les Règles de Durkheim un siècle après, Paris, L'Harmattan, 1996; M. Cherkaoui, Naissance d'une science sociale. La sociologie de Durkheim, Genève, Droz, 1998. 
tats dans les disciplines «dures». C'est ainsi que la topologie a été utilisée pour rendre compte des conflits sociaux, l'analyse des variations concomitantes pour expliquer les suicides, les analyses écologiques, contextuelles et dimensionnelles pour classer les ordres de préférence.

Le passage des données qualitatives (les plus nombreuses) ${ }^{49}$ à la quantification est assuré par l'acceptation de certains schémas de l'empirisme sensualiste et surtout par une manipulation tacite des outils de la quantification et du langage formel. Il suffit de se référer à l'échantillonnage tel qu'il est pratiqué en sociologie, pour en avoir la démonstration.

La théorie dit que pour fixer la grandeur de l'échantillon, il faut connaître le champ de la variance à mesurer. Si la variance de la variable est grande, le nombre des sujets de l'échantillon à déterminer devra être grand; si la variance est petite ce nombre devra être petit. Or cette variance en sociologie est toujours une estimation et c'est sur cette estimation élaborée sur la base d'une hypothèse invérifiable qu'on fixe la grandeur de l'échantillon et qu'on dit que la distribution est normale. Ensuite, on doit négliger le fait qu'il y a plusieurs variables, dont l'estimation de la variance devrait pourtant tenir compte. Ces variables, on les prend le plus souvent isolément, tout en sachant qu'elles ont deux ou plusieurs dimensions, et que cela est important pour la fixation du nombre des sujets. D'autre part, la théorie veut que les personnes interrogées soient choisies au hasard. Or cela se révèle presque toujours impossible dans la recherche sociologique. Pour avoir des échantillons représentatifs, on doit corriger les choix aléatoires par des choix raisonnés. Il est notoire que la théorie de l'estimation par l'intervalle de confiance n'est valable que dans le cas d'un sondage aléatoire, alors que tous les sondages sociologiques sont empiriques. En outre, l'échantillonnage par choix raisonné repose sur l'hypothèse que les variables de contrôle sont distribuées statistiquement comme les variables à analyser. Une telle hypothèse suppose l'étroite corrélation entre les variables de contrôle (sexe, âge, catégories socioprofessionnelles, habitat, religion, etc.) et le type de réponse donnée. Il est impossible de contrôler une telle hypothèse. De ce fait il n'y a aucun moyen pour évaluer la variabilité de l'estimation.

Nous avons là, grâce à la statistique, un outil important, mais dénué, en sociologie, de toute validité théorique et donc de légitimité pratique. On pourrait citer également les modèles déterministes pour l'analyse des processus sociaux; les modèles de simulation en tant que réalisation physique des modèles mathématiques, ainsi que certaines applications à la psychosociologie ou à la démographie sociale des modèles de simulation sans équivalents mathématiques directs. Et je laisse de côté les tables à trois dimensions, les croisements, les schémas de calcul des motivations, les panels et les preuves moyennant triangulation. Même lorsque des analyses à variables multiples sont possibles, peut-on dire que l'analyse des variables discrètes aide à saisir les systèmes entiers? L'agrégation des causes en systèmes occulte beaucoup de propriétés; elle n'arrive même pas à être la somme des parties et des relations causales spécifiques. L'étude des événements dont la fréquence est à la fois variable et mesurable n'assure pas la certitude d'identifier

Il me suffit de renvoyer à N. K. Denzin et Y. S. Lincoln. (eds.), Handbook of Qualitative Research, Newbury Park, Sage, 1994, et à C. Geertz, Local Knowledge, New York, Basic Books, 1983. 
les variables dont la régularité permettrait de les codifier en lois ou d'en élaborer une théorie.

Puisqu'il n'y a pas d'isomorphisme entre le monde physique et le monde social, les manipulations et les détournements sont permanents, les emprunts aux autres disciplines doivent subir des mutations, être transformés en métaphores ou en analogies ${ }^{50}$.

Avec cela je ne dis pas qu'en sociologie, et plus généralement dans les disciplines sociales, il n'y a pas d'arguments et de preuves quasi logiques. Mais ils restent des a priori car ils ne font pas appel à l'expérience. Toutefois chacun de ces arguments s'apparente à un principe logique, par exemple à l'identité et à la transitivité. Contrairement aux principes logiques de la démonstration, ils peuvent tous être réfutés. Les définitions (normatives, descriptives, condensées ou oratoires) sont constamment des identifications: elles établissent une identité entre le défini et le définissant de sorte qu'on peut substituer l'un à l'autre dans le discours sans pour autant en changer le sens. Pour ces raisons le chercheur en sciences sociales est ballotté entre le Deuten et l'Auslegen, entre l'interprétation en tant qu'imputation causale, en tant que distribution ou représentation, et l'interprétation en tant que signification herméneutique.

Les «classiques» de la sociologie offrent des exemplifications de ce qu'il vient d'être dit ici. Prenons le plus classique des tous les classiques, Max Weber. Pour lui les énoncés sociologiques, libérés des jugements de valeurs et de la croyance en l'existence des faits produits par l'expérience, sont vérifiables dans l'intersubjectivité. «Seules les régularités statistiques correspondent à un sens volontairement compréhensible de l'action sociale - et ce sont des types d'activité compréhensibles et donc des règles sociologiques ».

Comment a-t-il appliqué cette approche dans l'essai Ethique protestante et l'esprit du capitalisme (1904-1905)? Tout d'abord, sur la base d'un texte de Benjamin Franklin et des déclarations du banquier Jacob Fugger, Weber caractérise l'esprit du capitalisme en tant que recherche rationnelle et systématique du profit par l'exercice d'une profession et non par l'accumulation du capital. Ensuite, en se fondant notamment sur la Confession de foi de Westminster de 1647 , il définit l'éthique protestante comme l'accomplissement dans le monde des «devoirs correspondant à la place que l'existence assigne à l'individu dans la société, devoirs qui deviennent ainsi sa 'vocation'». Les liens entre l'ascétisme religieux et le développement de l'esprit du capitalisme seraient prouvés par les ouvrages issus de la pratique pastorale, notamment par les sermons et les livres du pasteur Richard Baxter, publiés dans la seconde moitié du XVII et au début du XVIII' siècle.

Etant donné qu'au XIX ${ }^{\mathrm{e}}$ siècle le capitalisme ne fait plus aucun recours à l'ascétisme religieux, Weber ajoute: «Resterait à élucider la façon dont l'ascétisme protestant a été a son tour influencé, dans son caractère et son devenir, par l'ensemble des conditions sociales, en particulier par les conditions économiques. Fût-il pétri de bonne volonté, l'homme moderne est incapable d'accorder aux idées religieuses l'importance qu'elles méritent pour les conduites, la culture et le

A ce propos il suffit de renvoyer à G. Busino, La place de la métaphore en sociologie, « Revue européenne des sciences sociales », XLI, 2003, n. 126, pp. 91-101. 
caractère national. Est-il nécessaire de protester que notre dessein n'est nullement de substituer à une interprétation causale exclusivement 'matérialiste', une interprétation causale exclusivement spiritualiste de la civilisation et de l'histoire qui ne serait pas moins unilatérale? Toutes deux appartiennent au domaine du possible; il n'en demeure pas moins que, dans la mesure où elles ne se bornent pas au rôle préparatoire, mais prétendent apporter des conclusions, l'une et l'autre servent aussi mal la vérité historique.»

Cette interprétation, basée sur des «preuves évanescentes», a provoquée aussitôt des critiques, des réserves et même des contestations pleines d'âpreté ${ }^{51}$. Parmi celles-ci, citons celles d'André Bieler ${ }^{52}$. Celui-ci, sur la base d'une documentation étendue, est arrivé à la conclusion que les preuves apportées par Weber se fondent sur des fausses corrélations entre les faits économiques et les conceptions religieuses des sectes puritaines. L'assimilation du puritanisme du XVIIIe siècle aux doctrines de Calvin, l'imputation au calvinisme de la primauté de la prédestination, de l'ascèse du travail, du mépris des plaisirs et de la vertu de l'épargne, prouveraient que Weber avait une maîtrise limitée des sources documentaires d'où ses méprises interprétatives allant jusqu'à la déformation.

De son côté, Herbert Lüthy ${ }^{53}$ affirme que les démonstrations wébériennes ne sont pas concluantes, que les comportements des hommes sont irréductibles à une causalité unique, que la chronologie «confirme mal et de façon singulièrement imprécise ce rôle déterminant de la Réforme», «qu'il est évidemment impossible d'expliquer le caractère et les réalisations de l'Occident même moderne [...] à partir d'un épisode unique de son passé récent, fût-il aussi virulent que le calvinisme ». Pour l'historien de Bâle, «Le mécanisme compensatoire mis en place par Max Weber est une subtile construction de l'esprit qui n'a pu fonctionner qu'exceptionnellement [...]; aucun des rapprochements tentés sur des points de doctrine ou d'éthique entre l'enseignement calvinien et l'économie capitaliste n'a vraiment résisté à la critique.»

51 Un excellent florilège de ces écrits a été proposé par Ph. Besnard, Protestantisme et capitalisme. La controverse post-weberienne, Paris, Colin, 1970. Cf. également K. Samuelsson, Economie et religion. Une critique de Max Weber. Introduction par D. C. Coleman, Paris, Mouton, 1971. Le texte de Die protestantische Ethik und der Geist des Kapitalismus se trouve in Gesammelte Aufsätze zur Religionssoziologie I, Tübingen, 1988, 9éd., pp. 1-236. Les traductions françaises sont peu fiables ainsi que vient de le démontrer J.-P. Grossein, A propos d' une nouvelle traduction de «L'Ethique protestante et l' esprit du capitalisme», in «Revue française de sociologie », 43-4, 2002, pp. 653-671.

52 A. Bieler, La pensée économique et sociale de Calvin, Genève, Georg, 1959.

53 H. Lüthy, «Calvinisme et Capitalisme»: après soixante ans de débats, «Revue européenne des sciences sociales», I, 1963, n. 2, pp. 5-35, article abrégé, amendé, enrichi et avec d'autres textes similaires, désormais in Le passé présent. Combats d'idées de Calvin à Rousseau, Monaco, Ed.du Rocher, 1965. J. Freund, Etudes sur Max Weber, Genève, Droz, 1990, pp. 139-164, discute posément ces thèses et affirme que «Lüthy entend prendre la défense de l'histoire contre une prétendue philosophie de l'histoire, dont la sociologie religieuse de Weber serait au moins en partie responsable.» Freund avait donné préalablement la liste des erreurs d'interprétations de Lüthy dans l'article intitulé Controverse sur Max Weber, «Preuves», n. 161, juillet 1964, pp. 85-87. Des remarques critiques ont été adressées à Lüthy et à Freund par G. Moreau, La controverse Julien Freund et Herbert Lüthy. A propos de Weber, in Historiographie de la Réforme, sous la direction de Ph. Joutard, Paris, Delachaux et Niestlé, 1977, pp. 307-321. 
L'essai de Martin Offenbacher, point de départ de la réflexion wébérienne, ne démontrerait, selon Lüthy, «que des variations marginales sur des chiffres absolus parfois infimes, dont l'insignifiance n'est camouflée que par leur traduction en pourcentage». En plus, Weber n'aurait «relevé et monté en épingle que le seul point qui venait appuyer ses conclusions formées d'avance». Weber décrirait «un certain calvinisme qui n'est guère celui de Calvin » et un capitalisme que personne ne reconnaît; il négligerait que «le siècle de la Réforme marque le début d'une longue régression». En effet, «l'Europe n'a plus atteint pendant plus de deux siècles le degré de développement et de structuration économique, ni cette vigueur d'initiatives individuelles, ni cette floraison de grands capitalistes d'allure royale, qu'elle avait connus au $\mathrm{XV}^{\mathrm{e}}$ et au début du $\mathrm{XVI} \mathrm{I}^{\mathrm{e}}$ siècle -au siècle des Fugger- à l'intérieur de la catholicité encore indivise.»

Toujours selon Lüthy, Weber a exclu de sa démonstration la Contre-Réforme, «réaction autoritaire contre l'esprit d'hérésie et d'insoumission» qui a «longuement bloqué le développement de la civilisation matérielle, technique et intellectuelles » des pays catholiques. Le sociologue allemand aurait négligé de «se poser la question de savoir si la Réforme [...] n’a pas simplement été, non la cause, mais le moindre obstacle à l'émancipation et à l'essor du capitalisme novateur». Il aurait ignoré « cette renaissance dans la conscience chrétienne de la Réforme de la tradition prophétique de l'Ancien Testament», «événement d'une importance incalculable dans l'histoire de l'esprit, qui touche tous les domaines de la vie privée et collective et affecte même les structures les plus profondes de la langue».

La conclusion de Lüthy est sévère. La méthode idéale typique est une nébuleuse, une fabrique d'approximations, d'abstractions généralisantes, tout à fait inadéquate à structurer une preuve. Les notions de prédestination calvinienne et de Beruf, ces types idéaux fondant l'ascèse protestante, «se sont révélées en fin de compte comme des noix creuses». "L'explication fondamentale du déplacement des activités économiques dynamiques consécutif aux bouleversements du schisme religieux, [il faut la chercher] non dans certaines conceptions dogmatiques du protestantisme ou du calvinisme, mais dans la réaction autoritaire de la Contre-Réforme qui a brisé l'évolution en cours et a imposé dans toute l'étendue de sa domination des structures hiérarchiques, cléricales et princières intrinsèquement hostiles au progrès intellectuel, technique et économique».

Lüthy oppose à Weber la thèse que l'agent actif du capitalisme moderne n'est pas «le calviniste torturé par l'incertitude de sa grâce aux yeux d'un Dieu tyrannique et arbitraire et cherchant absurdement à assurer son élection par le succès professionnel (construction indémontrable et presque saugrenue), mais le citoyen libre tel qu'il avait existé dans les républiques urbaines pieusement catholique d'Italie, de Flandres, de Haute Allemagne et de la Hanse d'avant la Réforme et tel qu'il ne pouvait plus exister désormais que dans les pays soustraits à la ContreRéforme».

Même en précisant que Weber utilise des interprétations postérieures d'un siècle et demi à celles de Calvin, que les notions de capitalisme et de protestantisme sont par lui considérées ambiguës, que pour saisir le réel en sa variété infinie il prône la construction de plusieurs idéaltypes, de diverses «rationalisations utopiques», qu'il n'y a pas un «rapport immédiat de causalité ou de fonction directe entre une doctrine religieuse et un comportement économique », il n'est 
reste pas moins que sa théorie n'est pas vérifiable, que son système de preuve n'arrive pas à prendre en compte le fait que des prêtres catholiques professaient des idées proches de celles du pasteur puritain Baxter et que dans certaines régions catholiques (par exemple, dans la Principauté ecclésiastique de Liège), dès la fin du XVI et jusqu'au XVII ${ }^{\mathrm{e}}$ siècle, le développement du capitalisme a été remarquable.

Corréler des phénomènes insuffisamment définis, isoler dans une série finie, d'une localité déterminée, pendant une courte période, un facteur particulier, puis en évaluer le degré de corrélation ou de covariance avec le processus général dont il fait partie, tout cela ne peut que produire des artefacts, jamais des preuves consistantes. Weber, certes, en est conscient, puisqu'il rappelle, à plusieurs reprises, que les rapports de causalité n'expliquent qu'une portion du réel, que la science du passé intégral et de la société global est irréalisable. Si Weber reste fidèle à l'idée que les preuves sont à la base de l'activité scientifique, il continue néanmoins à répéter que les phénomènes observables sont toujours donnés - à l'observation intuitive comme à l'observation scientifique - dans le temps et l'espace, et donc dans le cadre de leurs variations spatiales et temporelles. Cette thèse, qui s'applique à tout raisonnement explicatif sur des objets du monde, définit en effet, pour toutes les sciences et quel que soit leur objet, une unité logique du cadre cognitif et, en particulier, le fait que la phénoménalité des «faits » observables soit indissociable de leur historicité, c'est-à-dire de leurs variations historiques. Et, comme le même constat peut être fait sur les variations des faits de culture dans l'espace géographique, les deux propositions fondent ensemble, et cela dans toutes les sciences, la nécessité de recourir à la méthode comparative sous ses différentes pour construire des preuves. Puisque il y a une diversité des formes d'administration de la preuve dans les diverses sciences, Weber précise que l'historicité des faits entre lesquels le raisonnement scientifique institue des liens explicatifs n'est pas la même historicité lorsque (a) ce raisonnement peut s'appuyer sur un savoir nomologique déjà constitué, qui lui fournit, pour expliquer historiquement une succession d'états singuliers ( descriptibles, par exemple, dans l'histoire du «cosmos» ou de l'évolution biologique) un corpus de lois et principes universels tels ceux de la physique et de la chimie de la matière; et lorsque (b) le raisonnement scientifique n'a jamais pu constituer un tel corpus de référence au service de l'interprétation des singularités historiques étant donné qu'il n'a jamais pu conduire ses observations ni mener ses expériences selon la clause ceteris paribus. Cette «carence nomologique» des sciences sociales n'est ni fortuite ni momentanée; elle découle du type de récurrence ou de transformation des phénomènes historiques, ou écologiques), qui se présentent toujours comme des «constellations globales» constituées d'éléments étroitement associés dans une totalité et dotés chacun d'un sens se référant à cette totalité: de telles constellations ne peuvent jamais être complètement décomposées ou analysées en variables et encore moins en variables toutes quantifiables.

Grâce à cette distinction, Weber fait une nette séparation entre les sciences intégralement historiques et sciences de la nature qui restent, en leur fond, des sciences nomologiques, même lorsque ces dernières appliquent leurs connaissances nomologiques à la reconstitution d'une histoire. La distinction entre ces deux régimes scientifiques de la preuve est une distinction entre deux régimes épistémologiques différents d'administration de la preuve historique. Pour ces 
raisons l'histoire narrée par une science n'est pas la même histoire selon que peuvent y entrer les «faits virtuels» d'un paradigme théorique unifié ou qu'il s'agit de l'histoire d'une succession ou d'une cohabitation de constellations globales, jamais complètement analysables en systèmes de variations concomitantes entre leurs éléments ${ }^{54}$.

L'histoire de la sociologie nous offre des témoignages à foison sur l'indétermination de la notion de preuve, sur la répétabilité et la non-répétabilité, sur le fait que toutes les sciences empiriques ne sont pas expérimentales et qu'aucune ne se réduit à une démonstration déductive de ses conclusions empiriques.

Prenons, à titre d'un autre exemple, le cas de Middletown. A Study in America Culture (1929 et 1956) et de Middletown in Transition (1937) de Robert S. Lynd et de Helen Merrel Lynd. Ce couple de chercheurs s'installe, en 1925, à Muncie, dans l'Indiana, nommée pour la circonstance Middletown, et pendant 18 mois, avec une petite équipe de collaborateurs, ils récoltent des matériaux disparates (interviews, statistiques, coupures de journaux depuis 1890, témoignages, etc.) dans le but de décrire les problèmes de la petite ville, d'en dessiner l'identité. La méthode utilisée est celle de l'objective observer, de l'observation sans préjugés. Ils espèrent ainsi d'atteindre un degré élevé d'objectivité et en même temps d'arriver à corréler et rendre significatives des données nombreuses et hétérogènes. Les résultats des recherches sont consignés dans le volume intitulé Middletown. Les bouleversements provoqués par l'industrialisme (essor économique incontrôlé, diffusion de l'automobile, production de masse, chômage), par les changements dans les structures sociales traditionnelles (renforcement des inégalités, pauvreté, mutations des modèles culturels, apparition de nouvelles valeurs), ramènent les Lynd, en 1935, à Muncie. Les résultats de la nouvelle enquête paraissent en 1937. La théorie à la base des deux enquêtes est exposée, en 1939, dans Knowledge for What?

Au cœur de la recherche sur Muncie il y a un schéma à six entrées (gagner sa vie, constituer une famille, éduquer les enfants, emploi du temps libre, pratique religieuse, services pour la communauté) ainsi que la théorie des aires culturelles de Clark Wissler. Les grilles interprétatives, peu cohérentes, obligent les Lynd à concevoir Muncie comme une entité autonome, isolée du reste de la société américaine. Les classes, définies comme des groupements d'auto identification, de hiérarchies de revenu et de prestige, sont deux : le business-class (tous ceux qui exercent des activités non manuelles) et le working-class (les travailleurs manuels). Ces deux classes, réputées irréductibles aux rapports de production du système capitaliste, sont différentes à cause de leur manière de gagner la vie, de leur style de vie quotidien, des possibilités offertes aux enfants, de la disparité des intérêts économiques et de pouvoir. Les caractéristiques sociales du business class, constitutives de sa conscience unitaire, se manifestent dans la vie associative alors que le working class ne dispose d'aucune organisation ni de conscience communes.

${ }^{54}$ A ce propos voir les études réunies in M. Weber, Gesammelte Aufsätze zur Wissenschaftslehre, op. cit., mais aussi de J.-C. Passeron, L'espace wébérien du raisonnement comparatif, in M. Weber, Sociologie des religions. Textes réunis, traduits et présentés par J.-P. Grossein. Introduction de J.C.Passeron, Paris, Gallimard, pp. 1-49. 
Les Lynd constatent que l'évolution des mœurs, à Muncie, est brutale, que la production de masse y est intense. La tendance à résoudre les problèmes nouveaux avec des principes obsolètes, l'absence de dissidences et de critiques, la crise et les changements observés dans le domaine «gagner sa vie» sont expliqués à partir des théories de William Fielding Ogburn. L'indifférence à l'égard des personnes et l'incurie à l'endroit des institutions communautaires sont attribuées au rythme de développement de la culture matérielle, plus rapide de celui de la culture non matérielle. Le pourquoi de ce décalage reste mystérieux. Le capitalisme est retenu responsable de la crise sociale bien que son pouvoir n'ait été jamais identifié ni analysé. Les Lynd proclament l'incompatibilité de la propriété privée des moyens de production avec la démocratie sociale et pour cette raison ils prônent une réforme des institutions américaines. Ce vœu, excentrique et sans rapport avec la logique de la recherche, n'est que l'écho politique du «New Deal», de sa tentative de rationaliser l'économie, d'accélérer l'intégration socioéconomique, de maîtriser la crise sociale en contrôlant les concentrations économiques et l'évolution technique.

En 1935, dix ans après la première enquête, les Lynd retournent à Muncie pour y étudier les mutations intervenues au fil de la décennie. Ils utilisent le plan de la recherche de 1925. Les changements sont nombreux et marquants. La séparation entre les deux classes s'est accentuée, même au niveau des quartiers d'habitation. Le working class s'est différencié en strates. La strate supérieure des ouvriers spécialisés se distingue, économiquement, culturellement et racialement, des strates moyennes et inférieures. Elle est plus proche de la strate inférieure du business class, constituée d'employés et de petits entrepreneurs ruinés et devenus dépendants. La conscience d'appartenir à un groupe social, le besoin d'informations, le goût pour la lecture, le développement des organismes d'assistance sociale sont des faits nouveaux. Le business class a désormais une strate dominante, laquelle dispose du pouvoir, fixe les modèles de comportement et de consommation pour toute la communauté.

Les Lynd découvrent que la famille $X$ dispose de pouvoirs immenses, dont ils ne s'étaient pas aperçus en 1925. Les $X$ ont un véritable empire économique: de la finance à l'industrie et au commerce. Cette prépondérance économique leur donne un grand pouvoir social et politique, qu'ils utilisent pour garantir et développer leurs propres intérêts. Fournisseurs d'emplois, les $X$ imposent à la communauté un certain conformisme. Par l'entremise du crédit, ils contrôlent les commerçants, les artisans et les professions libérales. Grâce aux subsides et aux subventions, les $X$ influencent indirectement les écoles, les institutions culturelles, les médias, les églises, les partis politiques et l'administration publique. Tous dépendent du bon vouloir de la famille et le pouvoir de celle-ci se manifeste sous la forme d'un contrôle social des médias, des rôles dans le système productif et des processus de socialisation. Les critiques et les dissidents sont marginalisés, isolés, expulsés. Rien n'avive plus à Muncie les sentiments, les passions, les idées, d'où l'impossibilité de favoriser les changements et les mutations, d'y faire éclore un mouvement social. La sécurité y est devenue plus importante que l'innovation et les valeurs traditionnelles.

Dans Middletown in Transition les Lynd restent encore persuadés que cette situation est la résultante d'un conflit de valeurs plutôt que d'intérêts. Ils continuent à interpréter les rapports entre les groupes sociaux de façon empiriste. Les 
prévisions d'involution (par ailleurs aucune d'elles ne s'est réalisée) de la société américaine sont des supputations pessimistes. La conception dichotomique adoptée au cours de la première recherche laissa de côté maintes problématiques. Contrairement à la croyance ordinaire sur la puissance de la mobilité verticale ascendante et descendante dans la société américaine, les Lynd font entrevoir que la mobilité générationnelle est freinée par les difficultés, les obstacles, les résistances, les paralysies, tandis que l'intergénérationnelle, moins ardue, reste toutefois assez faible. Dans tous les cas, le poids de l'origine sociale est écrasant.

Les bouleversements qu'à partir de 1929 affectent la société américaine, contribuent à modifier la vision des classes sociales de Lynd. Le business class n'est plus qualifié de classe moyenne. Composée au sommet d'une couche de grands capitalistes, cette classe est de moins en moins attachée à la vie locale et de plus en plus intéressée à l'engagement national. Le conflit industriel, la compétition, le contrôle du pouvoir engendrent la domination de l'homme sur l'homme, d'une classe sur toutes les autres et empêchent la réalisation d'une société où la collaboration en vue du bien commun et de l'intérêt général sont possibles. Les Lynd sont désormais convaincus que le capitalisme est malfaisant.

Dans son livre Knowledge for what?, Robert Lynd, après une analyse de la crise de la famille, des rapports entre les sexes, de la question des jeunes, des vieux, du consumisme, du chaos des villes, de la corruption, de la culture, des dangers des guerres, affirme que la société moderne est déchirée par des contradictions et des conflits générés par le capitalisme. Pour les conjurer il faut remplacer la propriété privée des moyens de production par un autre mode de production des rapports économiques et sociaux soumis aux règles démocratiques. Dans cette perspective la sociologie a pour but d'identifier les conditionnements exercés par la structure économique sur la vie quotidienne des gens. Jusqu'ici, au nom de la neutralité et de l'objectivité les sciences sociales ou bien se sont réfugiées dans les abstractions et l'intellectualisme ou bien elles ont été les servantes des classes dominantes. Dans les deux cas, en acceptant la société telle qu'elle est, en considérant les institutions sociales immuables, les sciences sociales ont négligées les valeurs de la raison, du bien-être collectif, de la démocratie et l'importance des rapports interpersonnels. Elles ont fait l'apologie de l'ordre existant et ont contribué à le légitimer.

Dans les travaux des Lynd l'établissement des rapports de causalité est problématique, la sélection des antécédents et la détermination des possibilités suffisantes arbitraire tandis que la critique et l'engagement sociaux prennent le pas sur le prouver par preuves.

Les mêmes considérations peuvent valoir pour The Authoritarian Personality (1950) de T. W. Adorno et collaborateurs, célèbre enquête sur l'antisémitisme et sur la discrimination raciale, commencée en 1944 et achevée en $1949^{55}$. Ce travail prétend prouver que l'antisémitisme est une idéologie, celle du conservatisme politique, de la soumission à l'autorité, de l'autoritarisme à l'endroit des plus faibles, qu'il est une idéologie ethnocentrique, ayant une cohérence et une spécificité déterminées et définies, et que cette idéologie est en connexion avec la struc-

Pour un exposé exhaustif de cette recherche consulter M. Christie et M. Jahoda, Studies in the Scope and Method of "The Autoritarian Personality», Glencoe, Free Press, 1954, ainsi que M. Rokeach, The Open and Closed Mind, New York, Basic Books, 1960. 
ture autoritaire du caractère de la personne. L'antisémitisme est une rationalisation idéologique, une «projection » des comportements instinctuels, de modes de pensée exaltant aveuglément l'in-group et abaissant et décriant l'out-group.

L'enquête se base sur un échantillon de 2.099 sujets, tous des Américains de la classe moyenne, de race blanche, sans attaches ethniques et religieuses, choisis dans les Universités, les Syndicats et les Associations d'anciens combattants. Dans l'échantillon sont inclus 110 détenus du pénitencier Saint-Quentin et 128 patients d'un hôpital psychiatrique. Cet univers n'est nullement représentatif de la société étasunienne.

Aux 2.099 sujets sont administrés des questionnaires dans le but d'obtenir des informations sur leur collocation sociale objective, sur leur histoire personnelle, sur leur mentalité, sur leurs fantaisies, sur leur éthique et sur leur conception du monde. Les questions sont confectionnées de telle sorte que le sujet doit exprimer une approbation ou une désapprobation à propos d'énoncés dotés de valeurs implicites en suivant une échelle à trois dimensions positives et à trois dimensions négatives. Puis, avec les réponses obtenues on a construit quatre échelles et quatre paramètres à partir des évaluations des sujets : l'échelle de l'anti-sémitisme $(A-S)$; l'échelle de l'ethnocentrisme $(E)$; l'échelle du conservatisme politique et économique $(C P E)$ et l'échelle des tendances anti-démocratiques implicites $(F)$. Ces échelles sont construites de telle façon que les réponses affirmatives indiquent l'adhésion aux paramètres politiquement négatifs et donnent la mesure directe de l'anti-sémitisme, de l'ethnocentrisme, du conservatisme et des penchants antidémocratiques. Ensuite, on a classé les sujets en high scorers (le plus prédisposés à l'autoritarisme) et low scorers (les plus proches des tendances démocratiques).

Dans les résultats de l'échelle $E$ on a sélectionné 40 high scorers et 40 low scorers, qu'on a soumis à des longues interviews personnalisés et auxquels on a administré une série de questions projectives. Parmi les 110 détenus on a interviewé 8 high scorers (trois sont qualifiés de véritables fascistes) et 4 low scorers. Parmi les 128 malades mentaux, on a interviewé 28 high scorers et 31 low scorers et on a complété les entretiens à l'aide de leurs dossiers médicaux.

L'enquête utilise des élaborations statistiques mais aussi des techniques qualitatives (les 80 interviews). Selon les chercheurs, les données qualitatives vérifieraient les données quantitatives et vice-versa. Mais peut-on passer sans autre du dossier singulier, évalué au moyen de l'interview, aux données quantitatives, ou encore des moyennes statistiques aux cas individuels? Peut-on définir un type humain, faire son portrait psychologique et politique, sur la base d'un rapport supputé invariant (mais, en réalité, incertain, relatif, douteux, peut-être illusoire) entre une certaine vision du monde et une série de dynamiques psychologiques réputées typiques? La preuve de l'existence d'une structure autoritaire du caractère se concrétisant dans l'ethnocentrisme est inconsistante. Il n'est nullement prouvé l'existence d'un lien entre l'ethnocentrisme, attitude psychologique, ensemble de comportements évalués et mesurés par l'entremise de rationalisations foncièrement idéologiques, et l'autoritarisme conçu comme la composante naturelle, stable de la personnalité du sujet.

Dans cette enquête la personnalité autoritaire est à la fois la cause et la conséquence de comportements, de rationalisations, d'une idéologie, mais elle est aussi la personnification d'un penchant irrépressible au fascisme. En réduisant l'antisémite au fasciste, à personne autoritaire, l'antisémitisme et le fascisme deviennent 
des types psychologiques naturalisés, ils n'ont plus rien à voir avec la politique, l'économie et les institutions anti-démocratiques.

Les auteurs de cette enquête trouvent la preuve de la personnalité autoritaire dans les expériences préconscientes et inconscientes de l'enfance des sujets. La culture, les traditions familiales, les variables individuelles deviennent ainsi le compilateur incrémentiel des facteurs instinctuels et de l'appareillage biologique. Il n'est pas légitime d'étudier, avec des questionnaires et des tests administrés à un échantillon pas du tout bien formé, les causes de la structure des manières habituelles de sentir et de réagir qui distinguent un individu d'un autre. Il est impossible, avec ces méthodes, de déceler les relations entre le caractère, la structure sociale et les phénomènes politiques et sociaux. Dès lors l'explication naturaliste et psychologiste du fascisme ne peut être qu'une conséquence paradoxale et inéluctable.

Décrire certaines situations particulières n'amène pas à produire des explications. Des constats particuliers ne permettent pas d'élaborer des généralisations ou des discours universalisables. Les conditions de production des preuves, dont la vérification est impossible, font que la validité d'une démonstration n'a plus aucune valeur. Et lorsque le contexte particulariste est déterminant, les énoncés ont une valeur relative car les démonstrations et les preuves sont réductibles aux contextes d'énonciation et l'intelligibilité des significations idiopathiques fait abstraction des principes de l'universalisme logique.

Un effort considérable pour échapper aux paradoxes de cette recherche effectuée dans un contexte mais prétendant produire des propositions universelles, ou intersubjectivement communicables, est celui que Gunnar Myrdal a fait dans An American Dilemma. The Negro Problem and Modern Democracy (1944; nouvelle édition 1962) et dans Asian Drama. An Inquiry into the Poverty of Nations (1968). Cet auteur ayant résumé sa façon de faire recherche dans un petit livre, lequel se veut aussi un petit traité d'épistémologie pratique ${ }^{56}$, je me borner à constater que dans les deux enquêtes il y a des problèmes traités avec les méthodes quantitatives et d'autres avec les méthodes qualitatives. Les preuves des premiers sont vérifiables aisément, mais restent partiales, les autres résultent argumentées, plus ou moins rigoureusement, mais sont invérifiables. Les liens entre les deux approches sont fluctuants malgré la tentative de les expliciter dans les remarques conclusives. Myrdal est convaincu qu'une sociologie de la connaissance scientifique pourrait nous faire accepter l'idée qu'il n'y a pas de science dépourvue d'a priori. Sans ces derniers, indexés sur un contexte, il est difficile d'élaborer des théories, de poser des questions, enchaînées les unes aux autres, de produire des preuves à propos de la réalité sociale qu'on analyse. Mais alors l'universalité des énoncés existerait uniquement à l'intérieur du contexte de production? Leurs transferts dans d'autres contextes rendraient les énoncés particularistes, voire relatifs? Myrdal se borne à dire que les jugements de valeur sont inévitables dans la recherche sociale. En être conscients est le prix pour s'approcher de l'impartialité et de l'objectivité.

La recherche intitulée Commune en France. La Métamorphose de Plodémet (1967) d'Edgar Morin montre combien il est ardu, pénible, sur les terrains d'en-

s6 G. Myrdal, Objectivity in Social Research, London Duckworth, 1969. 
quêtes, de pratiquer l'impartialité, d'être conscients des difficultés dans les approches de l'objectivité, de produire des preuves valides au moyen de questionnaires, fiches médicales, entretiens et films. Et lorsque les chercheurs, en 2002, sont revenus dans cette commune du Finistère à fort taux de consanguinité, pour vérifier les résultats de la première enquête interdisciplinaire, ils se sont aperçus qu'ils n'avaient pas entrevu, au début des années '60, que les femmes et les jeunes étaient les agents secrets de la modernité et que leurs aspirations servaient déjà de moteur à l'ensemble du corps social. En d'autres termes, puisqu'ils n'avaient pas mesuré la prégnance de la langue et des traditions bretonnes, la pesée de la réalité socio-historique, les questions et les réponses furent biaisées. La méconnaissance de cette réalité a fait que les chercheurs ont parcouru «le terrain comme des voyageurs sans bagages $»^{57}$.

Une anthologie parue récemment fait l'inventaire de toutes les difficultés que nous rencontrons dans les enquêtes de terrain, et notamment dans les enquêtes qualitatives: les risques, pour l'inférence et la preuve, de l'observation participante; les inconvénients des procédures de codage et des critères d'évaluation; les difficultés pour contrôler les données; les incertitudes de l'approche réflexive, historique et comparée. L'auteur de cette anthologie nous rappelle en outre: «L'enquête de terrain doit toujours rendre des comptes à ses commanditaires, à ses sujets et à ses publics. Ses différents choix ne sont jamais purement et simplement de méthode: il lient par les engagements de la promesse ou de l'obligation, ils appellent des jugements sur les conséquences de ces actions. Sur le terrain plus qu'ailleurs, peut-être, la science ne va pas sans conscience. ${ }^{58} \mathrm{Il}$ faudrait ajouter que ces recherches, très souvent, confondent le commentaire social, l'idéologie, le discours critique sur la société, avec des théories ou l'enquête des sciences sociales.

L'histoire de la sociologie nous révèle que nous avons surtout contribué à la prolifération de théories, avec lesquelles nous avons créé les faits, et avec ces mêmes théories nous avons effectués les vérifications. Et nous avons baptisé ce mouvement circulaire, cette clôture, l'épreuve des faits, la vérification moyennant l'observation et le raisonnement.

Il est évident que les faits ne sont inscrits ni dans le monde qui nous entoure ni dans notre monde intérieur, et qu'il est illusoire d'imaginer pouvoir enregistrer les faits qui résultent toujours d'un ensemble d'opérations mentales d'organisation, de mise en relation et de complexification. Par ailleurs, en admettant un instant

A. Burguière, Bretons de Plozévet. Préface de R. Gessain, Paris, Flammarion, 1975, a fait un premier bilan des travaux suscités par cette enquête. Il a conclu, pp. 316-317, que la fragilité de cette recherche dérive de «la mise à l'écart de l'histoire», alors que celle-ci « est la seule parmi les sciences sociales à ne pas se définir par un objet mais par une dimension particulière de la réalité sociale: le temps ou plutôt 'l'historicité', dans laquelle elle se sent fondée à s'approprier les objets de toutes les autres sciences.»

58 L'enquête de terrain. Textes réunis, présentés et commentés par D. Céfaï, Paris, La Découverte, 2003, p. 470. A p. 607 nous lisons aussi: «La question des limites de l'honnêteté et de la transparence ne cesse de se poser. L'enquêteur doit-il être irréprochable et ne se permettre que de petits mensonges par omission, de façon à être clair avec ses informateurs et à ne pas entacher la réputation de sa profession? Faut-il renoncer à toutes investigation qui requiert de telles manipulations, d'autant que les informations recueillies peuvent l'être dans la plupart des cas par d'autres voies? La question peut prendre un tour explicitement politique.» 
qu'il y ait quelque part des faits donnés une fois pour toutes, ceux-ci ne pourront pas soutenir les déductions. Il faut loyalement reconnaître que la sociologie est obligée de recourir au non formel pour saisir la forme des faits et les processus, qu'elle nécessite d'une aide extérieure pour expliquer les systèmes formels dépourvus à la fois de sujets et de critères d'interprétation. Précisément pour ces raisons elle ne fait rien d'autre que produire du sens, des significations. Elle est le mode de production des raisons signifiantes des sociétés modernes. Science des significations sociales, des raisons symboliques, la sociologie se trouve enracinée dans les discours pratiques. Ses fonctions cognitives, opératoires et figuratives, d'explication et d'implication, dépendent essentiellement de la fonction symbolique générale qui est à l'origine de l'acquisition du langage et des signes collectifs. A travers la fonction symbolique et grâce à elle, l'expérience, comme action et construction progressive, accède aux conditions sociales d'existence. A travers elle et grâce à elle, tous les systèmes de représentation, verbale et non verbale, sont en condition de désigner des classes d'actions et d'objets, c'est-à-dire de générer les significations individuelles et collectives.

Pour toutes ces raisons, la preuve en sociologie n'est qu'une forme particulière d'argumentation, dont Jean-Claude Passeron, dans une série d'études remarquables, a tracé les contours ${ }^{59}$.

\section{HISTOIRE}

Dans cette discipline les discussions sur la notion de preuve n'ont pas été, au cours de ces dernières années, nombreuses. Est-ce que la théorie de la preuve concerne aussi l'histoire, cette activité intellectuelle qui vise à élaborer des récits, des «evidentia in narratione», des scénarios crédibles, des représentations de personnages et des situations, des enchaînements plausibles de «ce qu'a été »?

Pour Carlo Ginzburg la réponse ne fait aucun doute. Il accepte la thèse d'Arnaldo Momigliano d'après lequel l'histoire s'est constituée au croisement de la médecine, du droit et de la rhétorique. L'histoire examine des cas et des situations dans le but d'en trouver les causes naturelles et les expose ensuite selon les règles de la rhétorique. La preuve assure l'historien dans sa quête de la vérité. Les indices-effets permettent d'inférer les causes supposées. Ils sont les aspects nonintentionnels de la personnalité, non contrôlés consciemment, bien présents même si parfois ils demeurent cachés. La notion de preuve est à la base du métier de l'historien et de celui du juge. Entre les deux existerait une certaine proximité bien qu'ils utilisent des méthodes différentes et visent des objectifs divergents.

Pour l'historien il s'agit d'adapter le jugement aux faits constatés; pour le juge de prendre une décision, de la déclarer après un examen des faits et de la cristalliser en un jugement. Pour ce dernier les faits existent pour autant qu'ils sont nommés par la loi et qu'ils reçoivent d'elle une signification. Le fait vient après le

59 J.-Cl. Passeron, Le raisonnement sociologique. L' espace non-poppérien du raisonnement naturel, Paris, Nathan, 1991; Normes sociales et normes méthodologiques. A propos des styles d'intelligibilité dans les sciences sociales, «Revue européenne des sciences sociales», XXXIV, 1996, n. 104, pp. 11-55; Logique et schématique dans l'argumentation des sciences sociales, Ibid., XXXV, 1997, n. 107, pp. 169-196; Logique formelle, schématique et rhétorique, in L'argumentation. Preuve et persuasion, Paris, Ed. de l'EHESS, 2002, pp. 149-181. 
droit. La temporalité aussi n'est pas la même en histoire et en droit. En histoire il n'y a pas de prescription, mais uniquement des anachronismes. La logique du droit est binaire (vrai/faux, coupable/non coupable) et le jugement élaboré est un jugement de réalité, pas un jugement de valeur. Les marges d'incertitude introduisent les doutes alors que pour l'historien elles sont des occasions pour approfondir les questions et les tenir en suspens. Ils conçoivent et font intervenir le contexte mais pas de la même manière. Pour le juriste le contexte fournit une circonstance qui vient de l'extérieur atténuant ou aggravant une responsabilité. Pour l'historien il donne à l'action son sens et il se substitue à elle. Pour lui le contexte (social, politique, culturel, psychologique) est le «lieu de possibilités historiquement déterminées, sert à combler ce que les documents ne nous disent pas sur la vie d'une personne. Mais il s'agit là d'éventualités, pas de conséquences nécessaires; de conjectures, pas de faits avérés $»^{60}$.

Ginzburg reconnaît qu'il n'est pas toujours possible d'obtenir une preuve, c'est-à-dire «la possibilité de prouver que x a fait y; x pouvant désigner indifféremment le protagoniste, éventuellement anonyme, d'un événement historique ou le sujet impliqué dans une procédure pénale $»^{61}$. Il est convaincu que le travail de l'historien, bien qu'il ne puisse pas faire de l'expérimentation vu que les phénomènes étudiés sont irréversibles, arrive néanmoins à produire des résultats dans l'ordre des probabilités ${ }^{62}$.

Ces thèses soulèvent des nombreuses réserves et des doutes ainsi que l'ont révélé les discussions sur le «génocide des Arméniens », sur celui des Juifs ${ }^{63}$, et le

60 Ginzburg est très critique vis-à-vis de ceux qui disent que l'histoire vise à convaincre, que son but est l'efficacité et pas la vérité, que l'œuvre historique crée un monde textuel, autoréférentiel, lequel n'a aucun rapport démontrable avec les réalités extratextuelles dont il parle. Pour lui il y a divers types de narration dont les implications cognitives sont différentes. Chaque narration se base sur un code narratif. Celui-ci sélectionne certains aspects de la réalité, souligne certaines connexions, établit certaines hiérarchies. Les historiens étudient les rythmes de l'histoire, jamais les rythmes du récit historique, et ceci marque sa différence vis-à-vis de l'épopée, du roman et du film. Ginzburg est contraire à la théorie des représentations car elle transforme la source historique en source d'elle-même et ne prend pas en compte ce dont elle parle. Pour les approfondir le sujet, cf. L. Braudy, Narrative Form in History and Fiction: Hume, Fielding \& Gibbon, Princeton, NJ, Princeton University Press, 1970; H. White, Metahistory. The historical Imagination in Nineteenth-Century in Europe, Baltimore, John Hopkins University Press, 1973; L. Stone, The Revival of Narrative. Reflections on a New Old History, «Past and Present», n.85, November 1979, pp. 3-24; E.J.Hobsbawm, The Revival of Narrative. Some Comments, Ibid., n. 86, February 1980, pp. 2-8, à present dans On history, London, Abacus, 2002, pp. 246-253.

${ }_{61}$ Les citations sont tirées de C. Ginzburg, Il giudice e lo storico. Considerazioni in margine al processo Sofri, Torino, Einaudi, 1991 (trad. franç., Lagrasse, Verdier, 1997 ).

62 C. Ginzburg, Prove e possibilità. In margine a Il ritorno di Martin Guerre di Natalie Zemon Davis, Torino, Einaudi, 1984, pp. 131-152; Miti, emblemi, spie. Morfologia e storia, Torino, Einaudi, 1986 (trad. franç., Paris, Flammarion, 1989); Montrer et citer. La vérité de l' histoire, «Le débat », n. 56, septembre-octobre 1989, pp. 43-54, ainsi que Rapports de force. Histoire, rhétorique, preuve, Paris, Gallimard/Le Seuil, 2003. Sur cette approche historiographique, v. G. Busino, Causalisme, symétrie et réflexivité. Une lecture des travaux de Carlo Ginzburg, in L'acteur et ses raisons. Mélanges en l' honneur de Raymond Boudon, Paris, Puf, 2000, pp. 25-42.

63 Il suffit de renvoyer, par exemple, à Ailleurs, hier, autrement: connaissance et reconnaissance du génocide des Arméniens, in «Revue de la Shoa. Le monde juif», n. 177-178, janvier-août 2003, et à Devant l' histoire. Les documents de la controverse sur la singularité de l'extermination des Juifs par le régime nazi. Préface de L. Ferry. Introduction de J. Rovan, Paris, Cerf, 1988. 
procès Papon, à l'origine d'un important débat entre juristes, historiens et philosophes $^{64}$.

Certes, on peut admettre que l'histoire et les autres sciences sociales ont pour référent et objets communs le «cours historique du monde», cependant elles ne l'analysent pas de la même manière et les buts visés ne sont ni semblables ni analogues. Ces différences disciplinaires dépendent uniquement de la division du travail académique, des concepts spécifiques utilisés (diachronie/synchronie), des perspectives adoptées (particularisme/universalisme), des modalités descriptives ou explicatives (individualisme/holisme), des diversités des traditions intellectuelles qui rendent difficiles les transferts des acquis d'un domaine à l'autre? Je n'en suis pas sur. Mais au lieu de broder une fois de plus ces problématiques, examinons un cas désormais classique, les recherches consacrées à la «transition» et les approches adoptées par les différentes disciplines.

Les premières études sur la «transition», c'est-à-dire sur le passage et les transformations d'un type de société à un autre, remontent à l'époque d'Adam Smith, de David Ricardo et des philosophes du XVIII ${ }^{\mathrm{e}}$ siècle ${ }^{65}$. Ils ont été les premiers à décrire les effets et à expliquer les causes des bouleversements qui ont détruit les sociétés d'Ancien Régime et conduit à l'instauration des sociétés modernes. Karl Marx a repris, entre 1846 et 1876, ces études et les a élaborées en un schéma explicatif du fonctionnement et de l'évolution des différentes formations économico-sociales s'étant succédées dans le cours de l'histoire. Il a fait de même pour la correspondance entre les modes de production et les formes des rapports sociaux dans le but de dévoiler la loi générale qui gouverne la naissance, le développement, la transformation, le déclin, la mort des sociétés.

Depuis, les travaux des historiens, des économistes, des politologues et des sociologues sur la «transition» des sociétés traditionnelles aux sociétés industrielles, sur le passage d'une forme sociale à une autre, ont proliféré sans pour autant que les connaissances se soient véritablement accrues. Les travaux d'une historiographie à l'instrumentation statistico-mathématique sophistiquée, les études sociologiques et politologiques sur la modernisation, n'ont pas prouvé ni révélé les «logiques», les «causes », les « raisons » qui permettent la reproduction d'un système économico-social ni même les mécanismes qui favorisent la naissance d'une nouvelle organisation des rapports sociaux et l'aménagement de ceux-ci en une forme générale de société nouvelle.

Les explications de Marx sur le passage de la société féodale à la société industrielle capitaliste et les lois régissant le mouvement économique de la société

${ }^{64}$ A. Prost, Histoire, vérité, méthodes. Des structures argumentatives de l' histoire, «Le débat», $\mathrm{n}$. 92, 1996, pp. 127-140, et du même Argumentation historique et argumentation judiciaire, in L'argumentation. Preuve et persuasion, Paris, Ed. de l'EHESS, 2002, pp. 29-47; J.-M. Le Masson, Vérité historique, vérité judiciaire, «Droit et Société», 38, 1998, pp. 21-31; Y. Thomas, La vérité, le temps, le juge et l'historien, «Le débat», n. 102, novembre-décembre 1998, pp. 17-36; P. Ricoeur, La mémoire, l' histoire et l'oubli, Paris, Seuil, 2000. Une présentation synthétique de toute la problématique se trouve in O. Dumoulin, Le rôle social de l' historien. De la chaire au prétoire, Paris, Albin Michel, 2003.

65 Lire R. Romano, Le problème de la transition du féodalisme «at present» dans l' cuvre d'Adam Smith, «Revue européenne des sciences sociales », XXXIV, 1996, n. 106, pp. 17-24 et G. Busino, La permanence du passé. Questions d' histoire de la sociologie et d'épistémologie sociologique, Genève, Droz, 1986, pp.49-65. 
demeurent toujours la référence fondamentale, bien qu'elles négligent les questions de la transformation des régimes politiques, les idéologies et les structures de parenté. L'analyse des lois du mouvement économique n'étant réalisable que sur la longue durée, Marx doit classer les matériaux historiques dans un ordre chronologique qui va de la Révolution anglaise de 1640 à la Révolution française de 1789. Une attention particulière est réservée à l'Angleterre où la vie matérielle a été façonnée par les rapports capitalistes de production, notamment dans les domaines industriels et agricoles. Ensuite, la distinction entre la genèse des rapports capitalistes de production et le développement de ces mêmes rapports lui sert à élaborer une périodisation en trois époques.

La première se situe entre le $\mathrm{XIV}^{\mathrm{e}}$ et le $\mathrm{XV}^{\mathrm{e}}$ siècle et se caractérise par la crise de la production féodale et l'abolition du servage. A la fin de cette période il y a une augmentation du nombre des paysans libres, la prospérité des villes et des petits propriétaires terriens. Des changements radicaux commencent à affecter l'agriculture: de nouvelles formes de propriété foncière apparaissent ainsi que de nouvelles méthodes de production et la naissance des manufactures pour l'exportation. L'augmentation du nombre des travailleurs libres active le déclin et puis la disparition du système féodal.

La deuxième va du XVIe à la première moitié du XVIII ${ }^{\mathrm{e}}$ siècle. La révolution agricole se poursuit, la dépréciation des métaux précieux fait chuter le taux des salaires et le niveau de la rente foncière. L'augmentation du prix des marchandises enrichit les fermiers. Dans la même époque on observe le développement du régime colonial, du crédit, de la finance et l'avènement des premiers systèmes protectionnistes. Tous ces facteurs, agencés en diverses combinaisons, on les observe surtout en Angleterre, dès les années 1660, aux débuts de la révolution agricole. Les progrès de la révolution industrielle et du développement du machinisme sont si rapides et puissants, spécialement dès 1750, que les petits propriétaires terriens en sont les victimes. C'est ainsi qu'à partir de la fin du XVII ${ }^{\mathrm{e}}$ siècle, l'Angleterre supplante les Pays-Bas et devient le premier pays industriel du monde.

Pendant la troisième époque, de la fin du XVIII ${ }^{\mathrm{e}}$ au début du $\mathrm{XIX}^{\mathrm{e}}$ siècle, advient la structuration du mode de production capitaliste et la consolidation de sa base. Le machinisme et la grande industrie prospèrent; des formes spécifiques d'exploitation de la nature et d'accès aux ressources sont développées; des nouveaux modes d'organisation des processus de travail sont mis en place; la production et la redistribution des fruits du travail social. C'est précisément en cette période que les rapports de production deviennent aussi des rapports sociaux, qu'au mode de production vont correspondre des formes déterminées de parenté, de gouvernement, de pensée et de représentation du monde. Les rapports entre la structure et la superstructure composent une totalité organique et la production matérielle, dans la société, va dépendre de l'articulation des formes sociales avec le mode de production économique.

La démarche régressive permet de remonter de la structure des rapports de production capitalistes aux conditions historiques de leur genèse, de construire des généalogies, de sélectionner, dans l'ensemble des pratiques sociales et des événements du passé, les facteurs déterminant la situation présente. Ce procédé régressif s'accompagne à une approche développementale-constructiviste qui explicite la transition de la forme unique dominante vers les différentes formes de production du présent. 
Cela ne constitue pas une théorie du mode de production féodal, ni même une bonne formulation des lois économiques de la transformation ou de la dissolution des différentes formes de propriété et de production féodales. Tout au plus Marx nous fournit quelques indications sur la rente foncière, sur les formes économiques rattachées aux rapports de propriété féodaux, sur la manière dont ces rapports commencent à se défaire dès que la rente en travail et en marchandise se transforme en rente monétaire. La formulation marxienne met également en évidence le phénomène de l'accès des paysans à la propriété des terres, les modes de production basés sur la petite propriété, la transformation des droits féodaux en des rapports capitalistes dans la nouvelle agriculture. Ainsi l'analyse de l'accumulation primitive du capital dans le mode de production capitaliste est amorcée. Certes, la production de marchandises, la propriété privée, l'utilisation de l'argent comme capital, le travail salarié ont existé auparavant, cependant ils ne se sont étroitement imbriqués que dans les sociétés capitalistes. Pour cette raison l'étude de leur genèse est moins intéressante que celle des conditions de leur généralisation actuelle. L'analyse de la décomposition d'un mode de production et d'une formation économico-sociale, de la dissolution des anciens rapports et de la constitution des nouveaux, vise à démontrer que les contradictions entre la structure et la superstructure engendrent une nouvelle base matérielle et de nouveaux rapports sociaux.

Pour Marx la transition se réduit aux processus de formation d'une nouvelle totalité organique, dont les mécanismes fondamentaux sont essentiellement les relations sociales des rapports de production agricole qui rendent possible l'accumulation primitive du capital.

La plupart des historiens considèrent que cette théorie du mode de production capitaliste est en contradiction avec la succession des formes de la rente du travail salarié, du commerce et du capital financier. Convaincu que la succession historique des faits n'explique rien, que la description d'un nouveau mode de production, des facteurs qui le déterminent, l'analyse de la nouvelle organisation sociale et de la succession de ses diverses composantes ne rendent pas compte du passage d'une société à une autre, Marx se propose d'élucider préalablement les raisons ou les logiques qui régissent la succession observée. Cependant dans cette approche il n'y a aucune place pour le rôle des systèmes de parenté, pour les transformations des structures familiales lors de la genèse et de la consolidation des modes de production. Les facteurs susceptibles de rendre compte de la transformation des superstructures, des rapports non économiques et, en particulier, des formes de pensée élaborées et développées dans le mouvement de la croissance du capitalisme sont tous négligés. Pour Marx le rôle des systèmes d'idées et des mentalités dans la formation et dans la transformation des rapports sociaux sont insignifiants. Il ignore même les rapports entre le centre et la périphérie à l'intérieur d'un mode de production. Dès lors, nous n'avons pas une théorie bien formée de la transition d'une formation économico-sociale féodale à une formation économico-sociale bourgeoise ni la preuve que la révolution industrielle est à l'origine de la transition du féodalisme au capitalisme. Les concepts de croissance et de développement, de développement capitaliste et d'industrialisation, de processus d'industrialisation et de processus de modernisation, de modernisation et d'industrialisation, d'industrialisation et de capitalisme, utilisés de façon synonymique, sont imprécis, confus. 
Pour contourner ces difficultés des historiens ont décrété l'impossibilité de vérifier une théorie réaliste de la transition. Par contre, des économistes, des sociologues et des anthropologues ont tenté d'en affiner la cohérence et la rigueur.

Comme on le sait, les historiens ont étudié la nature du capitalisme dès le XIV ${ }^{\mathrm{e}}$ et jusqu'au XIX ${ }^{\mathrm{e}}$ siècle, et ont analysé ses particularités, ses différences et ses analogies d'une époque à l'autre, d'une région à l'autre. Ils se sont gardés jusqu'ici de tirer de leurs travaux une vision synthétique du phénomène. Fernand Braudel ${ }^{66}$ a brossé un tableau du capitalisme européen caractérisé par sa créativité excentrique, par son pouvoir de se soustraire aux conditionnements et aux uniformités, par une énergie quasi naturelle. Paul Bairoch, pour sa part, s'est efforcé de repérer et de reconstruire les facteurs à l'origine du développement (le progrès technique, les coefficients démographiques, la montée des prix, l'accumulation du capital). Pour lui la preuve de la genèse du capitalisme se trouve dans les progrès de l'agriculture antérieurs à ceux du secteur industriel ${ }^{67}$. L'agriculture, grâce aux effets directs et indirects de l'accroissement de la demande, aux interactions dérivant de ces effets, a mis en place les mécanismes de diffusion ou d'entraînement, des mécanismes fonctionnels grâce auxquels l'industrialisation a été réalisée. F. Braudel et I. Wallerstein ${ }^{68}$ donnent une phénoménologie du capitalisme et de l'économie-monde conçus comme force indispensable au mouvement sociétal, tandis que P.Bairoch de la description des principaux mécanismes économiques mis en place durant de la révolution industrielle, des rapports qui les unissent, des effets induits par leurs interactions, déduit la transformation de l'économie et de la société.

Les «social scientists» tantôt ont dénoncé les lacunes et les négligences du modèle marxiste tantôt ils ont essayé de le compléter ou de lui opposer d'autres modèles, tel celui de W. W. Rostow ${ }^{69}$. En quête d'une explication globale et unitaire, certains s'attachent à mettre en évidence les forces endogènes à l'origine de la transition, comme par exemple John Hicks ${ }^{70}$ qui les identifie au marché. D'autres se tournent vers les forces exogènes: A. O. Hirschman ${ }^{71}$ considère que c'est la mutation du système des valeurs éthiques et politiques qui a modifié le regard sur les phénomènes économiques et les activités de commerce et qui a fait de la poursuite de l'intérêt une règle de conduite mobilisatrice des passions. Le capitalisme serait parvenu à maîtriser les passions destructrices en les canalisant dans la cupidité et l'appât du gain, donc en tuant les passions, en détruisant le

${ }_{66}$ F. Braudel, Les ambitions de l' histoire. Edition établie et présentée par R.de Ayala et P. Braudel. Préface de M. Aymard, Paris, de Fallois, 1997.

67 P. Bairoch, Victoires et déboires. Histoire économique et sociale du monde du XVIe siècle à nos jours, Paris, Gallimard, 1997, 3 vols.

${ }_{68}$ I. Wallerstein, The Capitalist World-Economy, Paris, MSH, 1979; The Politic of the WorldEconomy, Paris, MSH, 1984; Le capitalisme historique, Paris, La Découverte, 1985.

69 W. W. Rostow, The Stages of Economic Growth, Cambridge, Cambridge University Press, 1960.

7o J. Hicks, A Theory of Economic History, Oxford, Clarendon Press, 1961, et Causality in Economics, London, Blackwell, 1979.

${ }^{71}$ A. O. Hirschman, Essays in Trespassing Economics to Politics and Beyond, Cambridge, Cambridge University Press, 1981, et Come complicare l' economia, Bologna, Il Mulino, 1988, mais surtout The Passions and the Interests. Political Arguments for Capitalism before its Triumph, Princeton, N.J., Princeton University Press, 1977. 
mystère et la magie, en supprimant l'indifférence. Et c'est bien cette mutation qui serait à l'origine du processus de transition de l'économie féodale à l'économie capitaliste.

Le moment le plus crucial de ces débats se situe au lendemain de la publication des études de Maurice Dobb ${ }^{72}$ sur le développement du capitalisme. Ce chercheur anglais s'est proposé de prouver que la thèse de Marx est vérifiée par tous les acquis de la recherche historique contemporaine sur la féodalité, sur les origines de la bourgeoisie, sur l'émergence du capital industriel, sur l'accumulation du capital, sur le mercantilisme, sur la croissance industrielle, sur la révolution industrielle et ses effets sur la période subséquente. Il conclut qu'il s'est produit une concentration de la propriété foncière entre les mains des seigneurs et de riches paysans aux dépens de la petite paysannerie. Ce processus de concentration d'une part et d'expropriation de l'autre a eu pour conséquences: (a) l'accumulation de capitaux dans les mains de la paysannerie, dont une part sera investit dans l'industrie; (b) la formation d'un marché intérieur: les paysans expropriés doivent acheter ce qu'auparavant ils produisaient eux-mêmes; de même, les paysans capitalistes doivent acheter les biens de consommation et les moyens de production; (c) les paysans expropriés constituent une main d'œuvre à la fois agricole et industrielle. La naissance de la société capitaliste est donc favorisée par la constitution du marché, par la formation d'une main d'œuvre abondante et par la disponibilité de capitaux. Ces éléments entraînent la différenciation entre la classe des propriétaires et celles des travailleurs, leur antagonisme, et par là la lutte des classes. Le mode de production capitaliste est ainsi constitué.

Ces thèses ont suscité des controverses innombrables. Alors que les travaux de Hicks et de Hirschman ont été jugés des exercices théoriques sans rapport avec l'histoire, les historiens se sont montrés plus prudents à l'égard des ceux de Dobb. Tout en reconnaissant que celui-ci maîtrise les résultats des enquêtes historiques, ses conclusions ont été jugées prématurées. Il généraliserait à l'excès des données locales et spécifiques. On lui a reproché d'avoir négligé l'Italie du XVe et du XVI ${ }^{\mathrm{e}}$ siècles et de réserver surtout de l'attention aux comparaisons des développements anglais et français. Dans sa construction à prétention globalisante trouvent une systématisation exclusivement les «réussites» et les «gagnants». Ceux qui n'ont pas «réussi», qui ont «pris du retard», qui n'ont pas voulu ou pas pu «réussir» sont absents. Dobb ignore également ceux qui avaient au départ une position favorable mais ont été dans l'impossibilité d'en profiter (c'est le cas de l'Italie du XVIe siècle) et ceux qui se sont révélés incapables de réaliser la transition (les Pays-Bas du XVII siècle).

Ce débat est décevant car il se borne à analyser le servage et l'organisation de la production de la propriété seigneuriale, à étudier l'articulation originaire entre producteurs directs et propriétaires terriens, à décrire le développement du commerce et de la production pour le marché. Le débat n'arrive pas à s'affranchir de la perspective exclusivement économique, ni à prendre en compte les «extravagances » de l'Italie de la Renaissance et des Pays-Bas du XVII' siècle. La vision

72 M. Dobb, The Transition from Feudalism to Capitalism, London, New Left Books, 1976 et Prelude to the Industrial Revolution, «Science and Society», Vol. XXVIII, n. 1, Winter 1964, pp. 31- 47. Voir aussi le recueil: M. Dobb, R. Hilton, E. Hobsbawm, A. Maczak, F. Mazzei, J. Merrington, A. Soboul, I. Wallerstein, Dal feudalismo al Capitalismo, Napoli, Liguori, 1986. 
économique du passage de la société traditionnelle à la société moderne, la recherche d'une explication généralisante basée sur les seuls facteurs économiques, le cas anglais considéré paradigmatique, tous les autres étant non pertinents, ont conditionné les débats. La transition en tant que phénomène essentiellement économique s'est manifestée en sa plénitude surtout en Angleterre, où la politique commerciale et coloniale en a été influencée. Ici les élites ont été attirées par le gain, ici s'est déployée une culture légitimant la réalisation de l'intérêt et les développements parallèles de l'économie rurale et d'une économie non agricole. Les analyses en termes de séries d'équilibres socio-économiques mouvants, donc des processus continus, linéaires, l'économie devenue le moteur de la transition et cette dernière l'indicateur premier de la transformation générale de la société. Pour qualifier le phénomène de révolution il a fallu donner de l'importance aux tensions, aux conflits, aux contradictions, aux antagonismes, identifier l'agent historique du changement, ignorer les résistances, les obstacles et les refus que les autres sujets de l'action historique ont opposé aux innovations et à la modernisation. C'est pourquoi, enfin, il a fallu gommer les discontinuités et ne mettre en évidence que les continuités, réduire les spécificités de chaque société, leurs formes particulières à des caractéristiques secondaires ou non pertinentes.

La transition en tant que processus économique implique l'autonomie de l'économique et ses déterminismes. Or ni les forces du marché, ni l'offre de maind'œuvre, ni l'adoption de nouvelles techniques, agricoles ou autres, ni les données monétaires, ne constituent des conditions nécessaires et suffisantes pour assurer le passage d'une forme de société à une autre. Le réductionnisme économique dissimule le fait que l'économie est toujours subordonnée aux facteurs socioculturels et politiques.

Une théorie de la transition bien formée devrait constater, pour prouver l'antériorité de la dimension économique dans le processus de transition, que le commerce et la finance sont bien à l'origine de l'accumulation primitive du capital, que les progrès agricoles et la croissance démographique ont donné de l'impulsion au développement protohistorique. Il faudrait aussi prouver que tous les grands centres urbains de l'époque préindustrielle ont perdu leurs rôles et leurs fonctions à cause d'un système économique et social qui, au cours des phases de transition, s'est situé entre le système urbain du commerce et de l'artisanat et celui du capitalisme industriel naissant.

Les économistes affirment qu'il existe des facteurs objectifs expliquant les retards persistants et les rattrapages. Les groupes humains mettent en œuvre face à des circonstances identiques des différentes solutions. Mais s'il est possible de faire un choix entre les différents facteurs, le système n'est ni mécanique ni nécessaire, il est une fonction de rapports sociaux à identifier. Si, par contre, l'industrialisation s'identifie avec le capitalisme, celui-ci est un système d'échanges basé sur le marché et la monnaie, ou bien il est un système d'accumulation du capital fixe au détriment du capital circulant. Pourquoi postuler que la maximisation est inhérente aux seuls comportements économiques capitalistes, alors qu'il est possible de maximiser n'importe quel type de comportement dans n'importe quel système économique à partir des seules valeurs ou des raisons signifiantes? Ce qui change dans l'économie capitaliste, ce sont les conditions et les contraintes, et donc les décisions, mais pas nécessairement les principes. Par exemple, l'accumulation et l'investissement peuvent se retrouver dans n'importe quel type de 
société. Ces deux éléments deviennent des fonctions déclenchantes si les milieux technologiques et socio-économiques se prêtent à un mode de production plus intense, qui valorise le profit monétaire. Il a fallu franchir un seuil décisif, celui de la transformation du travail humain en marchandise abstraite et cette marchandise en objet d'échanges, pour que l'échange prenne la forme qu'on lui connaît dans les sociétés capitalistes. Le passage d'un mode de production à un autre est un phénomène unique et irréversible. Les transformations qu'une société subit s'opèrent toujours à travers des équilibres précaires, multiples, variés et souvent aussi régressifs. Comment les identifier, les décrire et surtout les évaluer?

Cette problématique nous rappelle que: (A) Si le mode de production s'explique par rapport aux processus d'accumulation primitive alors il faut décrire et prouver prioritairement les mécanismes de l'accumulation et ses orientations, les mécanismes ayant conféré au capital une mobilité extrême et préciser si ces mécanismes proviennent des institutions financières. (B) Pour importante qu'elle soit, l'analyse des forces productives est reliée aux rapports que les groupes sociaux établissent entre eux en fonction de la propriété des moyens de production, de la répartition des ressources et des pratiques sociales. Mais comment étudier ces rapports dans les sociétés de l'époque préindustrielle? $(C)$ La participation à la détermination des pratiques sociales pose le problème de l'hégémonie, du pouvoir et de l'Etat, né de la désintégration de la société féodale, entre 1280 et 1360, produit des guerres, de l'impôt et de la laïcité. L'Etat aurait créé un marché unifié, mais il aurait aussi aboli le phénomène de la désertion d'un territoire par les populations, phénomène qui dans les sociétés sans Etat (comme, par exemple, chez les Nambikwara ou les Nuers) remplit la double fonction de désamorcer les conflits et de perpétuer un processus de migration et de transplantation continues ${ }^{73}$.

Dans les faits, les Etats des XVII et XVIII ${ }^{\mathrm{e}}$ siècles empêchèrent la désertion ainsi que la conversion au Protestantisme des individus et des groupes, mais jamais l'exode des capitaux, dont la mobilité était considérée, même par Montesquieu et par Adam Smith, comme étant de nature à prévenir les «grands coups d'autorité » des souverains. Dès lors, il faudrait déterminer également la fonction de l'Etat dans le processus de transition.

Les «nouveaux économistes» défendent un paradigme où l'explication économique est purifiée de l'antagonisme capital / travail et de la lutte de classe appliquée aux institutions. Inspiré par les «concepts économiques classiques», l'appareil conceptuel de ce paradigme est celui de la rationalité des choix humains. Douglass C. North ${ }^{74}$ est le plus célèbre théoricien de cette mouvance. Il attribue à chaque élément une autonomie relative dans le cadre d'un système d'interrelations, de sorte qu'il peut ramener toutes les explications sectorielles à une

Voir à ce propos le recueil d'études L'esprit des lois sauvages, Paris, Seuil, 1988, ainsi que le livre d'A. Testart, Les chasseurs-cueilleurs, ou l'origine de l'inégalité, Paris, Société d'Ethnographie, 1982.

74 D. C. North, The Rise of the Western World, Cambridge, Cambridge University Press, 1973; Structure and Change in Economic History, New York, Norton, 1982; Institutions, Institutional Change and Economic Performance, Cambridge, Cambridge University Press, 1990; Transaction Costs, Institutions and Economic Performance, San Francisco, CA, ICS, 1992. Lire sur l'oeuvre de ce prix Nobel: R. Giannetti et A. Baccini, Un Nobel a sorpresa, «Storia del pensiero economico», n. 26, n.s., 1993, pp. 3-22. 
seule cause, la cause des causes, et c'est l'explication ultime. La thèse principale est formulée ainsi: «La Révolution industrielle n'est pas la cause de la croissance, elle n'est qu'une des manifestations, l'un des révélateurs d'un phénomène nouveau, la croissance économique, dont les origines remontent à des temps anciens et au cours desquels s'est lentement construite la structure des droits de propriété. C'est cette structure qui a créé les conditions d'un fonctionnement social favorable à un meilleur développement des ressources à la société». Ce type de phénomène serait intervenu tout d'abord aux Pays-Bas, «la première nation européenne à se doter d'un système d'institutions et de droits de propriété permettant d'exploiter de façon efficace les motivations individuelles pour assurer l'orientation des capitaux et des énergies vers des activités socialement les plus utiles ». Cela a été possible grâce à la mise en place d'une technologie performante d'organisation des rapports humains, économiques et sociaux. Cette technologie dérive de l'octroi des droits de propriété, droits consécutifs à la fin du pacte féodal. Lorsque la noblesse n'est plus parvenue à assurer la sécurité et la justice, le système de servage qui conférait le droit à l'usufruit a perdu sa raison d'être. L'équilibre a été rompu par la croissance démographique. La fin de l'usufruit féodal et du servage va donner naissance, en trois étapes, aux formes

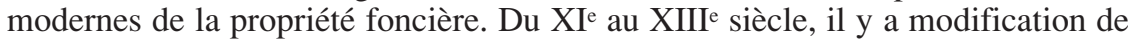
l'économie du système de servage, car la terre est rare et le travail abondant. Les paysans acquièrent la pleine propriété de leur force-travail, c'est-à-dire qu'ils sont libérés de leurs obligations corvéables contre le paiement d'une taxe annuelle. Dès ce moment, le paysan demande au seigneur la location du domaine qu'auparavant il cultivait en ne payant que l'usufruit des produits du domaine seigneurial. Le droit héréditaire à l'usufruit, représenté par le travail gratuit du serf, est remplacé par la rente, sorte d'obligation monétaire contractuelle enracinée dans un droit d'exploitation foncière. Il ne s'agit pas encore de la rémunération d'un droit de propriété, mais c'est déjà sa genèse. Avec le temps, lentement, les rapports féodaux de suzeraineté se relâchent et libèrent la propriété de la forcetravail par le truchement de la taxe; ils délivrent également des obligations personnelles moyennant le paiement d'une somme forfaitaire. L'individu acquiert ainsi le droit de propriété sur sa force de travail personnelle, tandis que le vassal se dégage de ses obligations en versant un «impôt» à son suzerain. C'est ainsi que se met peu à peu en place un véritable droit de propriété seigneurial.

Dès la seconde moitié du XIII siècle la raréfaction des terres et la hausse des prix agricoles accélèrent le mouvement. L'économie nouvelle démantèle l'organisation basée sur le servage et ruine le pacte féodal. Bien qu'elle soit décimée par les famines et les épidémies, la population croît plus rapidement que la production. Les termes de l'échange favorisent davantage les paysans que les titulaires des droits féodaux. La durée des baux s'allonge. La précarité de la location disparaît lorsque le seigneur reconnaît la transmissibilité du droit de location d'une génération à l'autre moyennant le paiement d'une solde à chaque succession. Lorsque s'accentue au XVI' siècle le cycle malthusien d'expansion et que l'inflation en souligne la force, les termes de l'échange favorisent la montée des loyers fonciers au profit de la noblesse. La baisse des salaires fait disparaître les derniers résidus du servage, désormais improductifs, puisque la main d'œuvre salariée est beaucoup moins chère que les serfs. C'est la fin des institutions féodales, la naissance de la propriété foncière et aussi de la propriété individuelle. 
Pour combattre les effets de la dépression malthusienne du XVIIe siècle, il faut augmenter le rendement des terres. Ce qui aboutira à la révolution agricole, prélude à la révolution industrielle. Les innovations technologiques ont été réalisables grâce aux droits de propriété. La définition rigide de la structure juridique du système foncier, dont le mouvement des enclosures est l'aspect le plus significatif, stimule les intérêts individuels et collectifs et incite à des choix dont la rationalité économique se révélera plus performante, donc plus rentable.

Le modèle proposé par North est une version nouvelle du modèle classique de Malthus et de Ricardo. Les variations de la population, les rendements croissants de la population agricole, le mouvement des prix relatifs en constituent les pièces maîtresses. L'accroissement de la population, et donc des besoins alimentaires, oblige à mettre en culture de nouvelles terres et à augmenter le rendement de celles qui le sont déjà. Mais les salaires diminuent puisque la main d'œuvre est abondante. Les prix des produits agricoles, en revanche, augmentent. Ce seront les famines et les épidémies qui rétabliront l'équilibre en diminuant la population, et donc la demande en produits alimentaires. Les prix suivront le même mouvement descendant. La main d'œuvre étant devenue rare, les salaires augmentent, alors que les ventes et les profits diminuent. La nouvelle répartition du revenu favorise l'augmentation de la population, et le cycle peut recommencer. Selon North, la logique du cycle a été rompue par l'introduction d'institutions nouvelles permettant une organisation économique plus efficiente, c'est-à-dire une meilleure utilisation du mouvement des prix relatifs. Les possibilités de changement sont par conséquent liées au système des prix, indicateur des raretés relatives de nature physique et donc générateur de comportements de résistance anticyclique. L'état des prix permet donc d'apprécier les situations et d'élaborer des réponses toujours plus adéquates. Lorsque les institutions favorisent ce processus, la transition pourra s'opérer. En d'autres termes, le passage d'une société à l'autre est assuré par le mode d'organisation des rapports sociaux. Selon que les institutions réglant l'organisation de ces rapports favorisent les choix en maximisant l'intérêt individuel et collectif, les décisions ultimes seront plus performantes. Parmi toutes les technologies mises en œuvre au service de la «performance» des rapports humains et sociaux, ce sont les droits de propriété qui ont permis aux sociétés occidentales de gérer au mieux le passage des sociétés traditionnelles aux sociétés industrielles. Selon Harold Demsetz «les droits de propriété permettent aux individus de savoir a priori ce qu'ils peuvent raisonnablement espérer obtenir dans leurs rapports avec les autres membres de la communauté. Les anticipations se matérialisent par les lois, les coutumes et les mœurs d'une société. Détenir des droits, c'est avoir l'accord des autres membres de la communauté pour agir d'une certaine manière et attendre de la société qu'elle interdise à autrui d'interférer avec ses propres activités, à la condition qu'elles ne soient pas prohibées. Les droits de propriété permettent à leur détenteur de faire du bien ou du tort aux autres membres de la société, mais pas n'importe quel bien, pas n'importe quel tort ${ }^{75}$.

Les droits de propriété définissent la manière dont les individus peuvent soit tirer un profit de certaines activités soit être pénalisés à cause d'elles. Ces droits

\footnotetext{
H. Demsetz, Towards a Theory of Propriety Rights, «American Economic Review », 57, 1967, pp.
} 347-359. 
déterminent par conséquent qui doit payer pour modifier les actions d'autrui. La reconnaissance de ces droits conduit à établir une relation assez étroite entre les droits de propriété et le contexte extérieur. Dans ces conditions le rôle et la place des organisations et des institutions qui permettent la transition et déterminent la performance de l'économie sont essentiels. Cette théorie néglige toutefois le fait que toute transition est en dernière analyse la solution d'un conflit et que dans un conflit nul ne peut être gagnant sans laisser derrière lui un perdant. Le marché, mécanisme indispensable au maintien des droits de propriété, n'est pas neutre, il est plutôt le reflet, la caisse de résonance des valeurs collectives relativement à la supériorité des droits de propriété.

Les économistes ont développé les concepts d'utilité, de préférence et de demande révélée pour expliquer le fonctionnement d'un système économique indépendamment des sentiments de ses participants. Sur le marché, le bien-être de tous serait maximisé sans que personne n'ait à se préoccuper préalablement du bien-être de son voisin. Or, la «main invisible» d'Adam Smith n'est pas un don spontané de la nature, mais bien le produit d'un ensemble de choix collectifs en faveur du droit de propriété, assorti d'un certain nombre d'autorestrictions qui en sont le corollaire. En effet, le droit de propriété exercé par un individu sur un bien en limite la jouissance pour autrui. Les ressources étant limitées et les désirs individuels infinis, la rareté relève donc d'un phénomène social et non d'un fait de nature. Il en découle que l'interdépendance des individus persiste, même lorsque le système des droits de propriété ferait croire à la liberté individuelle. Nous sommes privés, en quelque sorte, de ce que possèdent les autres, et réciproquement. Dans le même ordre d'idées, l'ensemble des possibilités offertes à un individu est vu comme un don particulier, limité d'une certaine façon par la collectivité, c'est-à-dire par les interdictions légales. Or, on peut décrire toutes les possibilités offertes à un individu d'un point de vue statistique, mais à condition de les isoler, de les séparer du monde dans lequel elles s'insèrent. Ce qui est irréel, puisque chaque individu vit dans un environnement social, dans un système d'actions qui comprend également les autres ${ }^{76}$. L'interdépendance des individus, l'ensemble des possibilités, le pouvoir, le mode de transaction montrent que le droit de propriété relève d'un consensus social sur ce qui fonde le pouvoir d'un individu sur un autre. Ce consensus tacite est un choix public et il serait naïf de le regarder comme un don de la nature ou l'effet d'une main invisible. En plus, l'explication par l'économie est téléologique et anachronique. Elle est finalisée sur la base d'une fin-valeur d'aujourd'hui: l'économie de marché. Considérant qu'il s'agit du stade final, elle envisage le passé comme une préparation à l'économie du marché capitaliste des biens, des services ainsi que des facteurs les rendant possibles (capital, force-travail, terre). En bref, le passé est reconstruit sur la base des exigences de fondation de la théorie néo-classique contemporaine, il est reconstruit et expliqué uniquement en fonction du présent.

Les limites de ce modèle sont évidentes: trop d'hypothèses simplificatrices (par ex., le changement institutionnel explique l'évolution des sociétés), un usage immodéré des tests économétriques, l'impossibilité de mettre en relation des

Cf. G. Berthoud \& G. Busino, Pratiques sociales et théories. Les discordes des universitaires, Genève, Droz, 1995, pp. 41-94. 
concepts invariants avec une réalité en perpétuel changement, une croyance utilitariste dans les mécanismes du marché, dans le profit individuel et dans les droits de propriété comme facteurs essentiels de l'évolution sociale de la société féodale.

L'exemple de la «transition» montre combien grande est la distance séparant les historiens, les économistes, les juristes et les sociologues à propos du «prouver par preuve $»^{77}$.

\section{0. ÉPILOGUE}

La pratique des sciences de l'homme et de la société est caractérisée par une pluralité de constructions théoriques, des approches différentes, des multiples cadres d'analyse, par des façons d'élaborer des preuves relativement indépendantes des théories en tant que systèmes conceptuels ou ensembles propositionnels. Le pluralisme théorique engendre un pluralisme des faits. L'accès aux preuves empiriques est problématique. L'utilisation de la langue naturelle favorise peu l'épuration des constructions théoriques et non théoriques, rend la cumulativité très compliquée et complexe et l'existence des paradigmes aléatoire. Bien que les théories ne soient pas régulièrement formées par la logique, bien que les prédictions ne soient pas exactes, bien que normalement les preuves ne recueillissent pas l'assentiment général de la communauté des chercheurs, il faut reconnaître cependant que celle-ci accepte de façon unanime les principes rationnels régissant l'activité de recherche. Il est vrai aussi que dans certaines disciplines des sciences humaines l'accord entre chercheurs, l'acceptation de certains paradigmes, l'utilisation de certaines techniques et méthodes, sont plus consensuels ou pacifiques, alors qu'ailleurs sont très conflictuels.

Passeron, pour expliquer ce pluralisme, a proposé la distinction entre les théories sociologiques et la théorie de la connaissance sociologique et a insisté sur la dimension historique des sciences sociales. Il a présupposé qu'il est possible de distinguer les informations empiriques (vérifiables ou vérifiées), les effets de connaissance (les opérations de reconceptualisation effectuées sur les informations empiriques et grâce auxquelles on produit d'autres informations vérifiables) et les effets d'intelligibilité (la reconstruction systématique des effets de connaissance dans une théorie). Il a insisté sur le fait que ces sciences ne sauraient établir des lois nomologiques, qu'elles doivent se servir des langues naturelles et peu des langages formelles, que l'intelligibilité des systèmes sociaux, toujours en devenir, ne peut pas faire l'économie de la reconstruction de leur genèse ${ }^{78}$. Bouvier, de son côté, constaté que l'histoire prend la forme d'un dialogue sans fin entre le présent

77 Sur les rapports entre les historiens et les économistes lire aussi E. Hobsbawm, On History, London, Abacus, 2002, pp. 124-163.

78 J.-Cl. Passeron, De la pluralité théorique en sociologie. Théorie de la connaissance sociologique et théories sociologiques, «Revue européenne des sciences sociales», XXXII, 1994, n. 99, pp. 71116. Cette distinction a été analysée de près par J.-B. Grize, Commentaire sur le pluralisme théorique, Ibid., pp. 117-121 et par J.-L. Fabiani, Epistémologie régionale ou épistémologie franciscaine? La théorie de la connaissance sociologique face à la pluralité des modes de conceptualisation dans les sciences sociales, Ibid., pp. 123-146. Un point de vue plus éclectique est défendu par J.-M. Berthelot, Programmes, paradigmes, disciplines : pluralité et unité des sciences sociales, in Epistémologie des sciences sociales, op. cit., pp. 457-519. 
et le passé, propose une perspective polyphonique, un dialogisme, qu'il oppose à la perspective homophonique où un système a de sens dans la mesure où il prépare l'avènement du système final ${ }^{79}$. L'un et l'autre reconnaissent que les sciences humaines sont plurielles, que leurs cadres d'analyse sont disparates, que leurs démonstrations ne descendent pas d'un ensemble d'axiomes posés une fois pour toutes, que leurs preuves ne répondent à aucun des critères posés par les épistémologues des sciences.

L'acceptation d'une telle thèse implique l'adhésion à une forme de relativisme et l'abandon de la sociologie en tant que discipline scientifique? Je ne le crois pas.

Si nous définissons la société par ces conditions d'existence, par son travail, par ses conflits, par ses réseaux de significations, par son système signifiant, par ses représentations, il est inutile de distinguer la sociologie de l'histoire, puisque ces disciplines s'occupent précisément de l'ensemble des ensembles, selon la belle formule de Fernand Braudel. Dès lors, fusion, compénétration de l'histoire et de la sociologie pour cause de complexité de la réalité sociale? Peut-être, mais comme le caractère complexe des phénomènes dépend essentiellement de la façon dont nous les regardons, cela revient à dire qu'il faudrait inventer un nouveau type de discours.

Jean Piaget n'aimait pas qu'on lui parle des questions de «juridiction disciplinaire». En revanche avec fermeté il disait: si la société est l'ensemble des ensembles des rapports sociaux, si les rapports sociaux modifient à la fois la nature et nos comportements, si la société est l'entrecroisement de séries de générations pesant sur les suivantes par l'intermédiaire des signes, des valeurs et des règles, c'est-à-dire du langage, des échanges et des normes collectives logiques et prélogiques, il faut faire de la sociologie et de l'histoire.

Bien entendu, sa conception de la contrainte exercée par le tout social sur les individus, qui prend les formes de la solidarité coopérative dans nos sociétés, alors qu'elle demeure limitée au conformisme obligatoire dans les sociétés dites primitives, fait problème. Piaget nous a cependant aidé à ouvrir les yeux sur les recherches qui liquident l'opposition sociologie-histoire ainsi que l'opposition société-nature. Il nous a poussé à lire les travaux visant à fonder, sur la base de la biologie, de l'éthologie, de l'écologie et de tant d'autres disciplines, une véritable science de l'homme, un véritable savoir anthropologique.

Certes, nous n'avons plus aucune certitude absolue sur l'existence d'un ordre social autonome. Le sociologisme en général et les paradigmes déterministes en particulier ont évolué vers un néo-durkheimisme aux contours de moins en moins exclusifs. Un nouveau souffle a été donné aux paradigmes interactionnistes, au déploiement des catégories de choix, d'intention, d'action, au dépérissement des doctrines utilitaristes et hédonistes. Les interrogations, les contestations, les doutes, les quêtes revigorent le pluralisme et mettent en question les modèles obsolètes. La sociologie réflexive des sciences sociales fait entrecroiser des problèmes généraux, aide à déceler les mécanismes communs, leurs fonctionnements, à comprendre les manières adoptées par les «social scientists » pour saisir les expériences sociales, pour les exprimer selon des façons différentes.

A. Bouvier, Un modèle polyphonique en épistémologie sociale. Croyances individuelles, pluralité des voix et consensus en matière scientifique, «Revue européenne des sciences sociales», XL, 2002 , n. 124 , pp. 39-58. 
Nous savons qu'il est dangereux de réduire les différences à l'identité. Nous savons aussi que la raison à l'œuvre dans les sciences sociales suit des règles informulables en termes rigoureusement logicomathématiques. Il en va de même, d'ailleurs, pour les sciences dites exactes lorsqu'elles formulent leurs discours en une langue naturelle. Chaïm Perelman, avec ses remarquables études sur la logique de l'argumentation, a montré combien il est difficile de faire appel à la preuve expérimentale ou à la preuve par calcul lorsqu'il s'agit de faits sociaux, de délibérations et discussions, de décision et de choix. La raison délibérative s'enracine dans la pensée argumentative, et, à l'opposé de la pensée démonstrative, recourt obligatoirement à des méthodes non déductives et non formelles. Le raisonnement des sciences sociales relève davantage de l'argumentation que de la démonstration pratiquée dans les systèmes axiomatiques formalisés. Même les efforts d'un Georg $\mathrm{H}$. von Wrigt visant à développer une théorie générale des modalités, axée sur les concepts modaux de «nécessaire», «possible», «impossible», «contingent», ainsi que sur les concepts déontiques d' «obligatoire», «permis », «défendu», «indifférent», tout en laissant une bonne place à l'agir intentionnel, ont été néanmoins incapables de nous faire comprendre la nature ontologique des normes sociales, d'expliquer comment on peut en établir l'existence et la validité. En effet, von Wright, pour montrer comment nous passons des intentions à l'action, du rapport entre celles-ci aux voies à suivre pour l'expliciter, doit recourir aux catégories téléologiques et ensuite les rapporter à l'explication causale. Les résultats sont évidemment maigres.

Je ne parlerai pas ici de Vilfredo Pareto, de sa théorie des argumentations impures ou imparfaites et de sa théorie, très contrastée, de la pensée scientifique et de la rhétorique politique, et selon lequel le problème essentiel des sciences sociales n'est pas celui de la rationalité mais de la croyance. Par contre, ce qu'il importe d'affirmer ici c'est que Jean Piaget, en désignant comme forme suprême d'intelligence la raison, la pensée abstraite telle qu'elle se manifeste dans les mathématiques, a choisi a priori pour la science un mode de rationalité. Ce choix me paraît arbitraire. Il passe sous silence toutes les autres rationalités à l'œuvre dans nos conduites ainsi que dans les religions, les mythes, les imaginaires, etc. Certes, dans les mythes et dans l'imaginaire on peut dégager des structures et à ces structures donner des formes logicomathématiques. En la matière, René Thom, avec ses modèles mathématiques de la morphogenèse, avec sa typologie des langues naturelles et des régimes symboliques, a ouvert des pistes sur lesquelles, en vérité, personne jusqu'ici n'a osé s'aventurer. Pourquoi? Parce que les formes de Thom ne peuvent s'élaborer d'une façon rigoureuse qu'à l'intérieur d'un système formalisé supposant un langage artificiel et immuable, ou présupposant le paradigme booléen. Or, l'écart entre l'algèbre de Boole et la pensée naturelle est grand. Par ailleurs, la pensée naturelle n'est jamais foncièrement irrationnelle. En disant cela, je n'entends point affirmer l'existence de plusieurs raisons; j'affirme seulement que nous appréhendons la réalité sociale, que nous la produisons et la reproduisons à partir de points de vue et des rationalités très divers, d'où la saisie d'aspects différents, de points de vue apparemment irréductibles, de singularités, de vérités valables par rapport à des territoires locaux.

Pourquoi ne pas le dire? La science, au sens où les corpus des connaissances ont été élaborés, puis perçu en Occident depuis Galilée, n'est plus aujourd'hui qu'un savoir parmi d'autres, une partie d'un savoir plus général. La science n'est 
qu'un pan du polyèdre de la connaissance. Ses progrès sont considérables et ils sont assurés grâce à la méthode des approximations successives, grâce à des modèles partiels et partiaux, constamment dépassés ou généralisés. Ce qu'elle néglige ou n'arrive pas encore à prendre en compte n'en existe pas moins et demeure en tout cas accessible par d'autres voies ou en d'autres moments.

Etant donné qu'un système axiomatique formalisé est dépourvu à la fois de sujets et de critères pour son interprétation, pour donner des interprétations des formes pures il faut faire appel à des propriétés extérieures aux formes, donc à des éléments non formels. Dès lors, nous pouvons élaborer une multitude d'interprétations du même système formel, et pourtant nous ne pouvons en utiliser qu'une et une seule à la fois. Il est évident que lorsqu'il s'agit d'interpréter le devenir, le développement, la genèse, les décisions individuelles ou collectives, les choix publics et privés, les comportements de la vie quotidienne ou les situations sociohistoriques, cette univocité est inadéquate, voire futile. En effet, la formalisation est impuissante à saisir le sens, les significations, puisqu'ils lui demeurent étrangers. Dans certains cas, elle est une condition nécessaire, quoique jamais suffisante, pour dégager le sens, car celui-ci n'est saisissable que sous une certaine forme. Néanmoins, la formalisation, pour atteindre ce sens et les significations attenantes, doit les connaître au préalable, les avoir posés au départ, ou tout au moins pouvoir les déduire. Cela n'est guère possible, parce que l'indétermination, l'ambiguiité, la polyvalence, propriétés constitutives du sens, restent idiosyncrasiques au système formel; parce que celui-ci est assujetti à une organisation conceptuelle atemporelle, indifférente aux pratiques symboliques et aux situations historiques et sociales.

Or, les sciences sociales sont essentiellement production de sens, de significations; elles sont le mode de production des raisons signifiantes des sociétés modernes. De la construction de la réalité sociale à la production d'informations, de la description des processus sociaux à l'analyse des systèmes sociaux, les sciences sociales attribuent, distribuent, représentent, grâce à la construction de la réalité sociale et à son interprétation, des sens et des significations. Ces derniers constituent, ensuite, les enjeux des conflits entre acteurs sociaux, les fondements des divisions, les raisons des luttes. Les sciences sociales sont les sciences des significations sociales, des raisons symboliques, des raisons signifiantes; elles sont là où s'élaborent des significations, avec les producteurs de sens, devant eux ou à côté d'eux, parties intégrantes des structures non significatives qui organisent les moyens dans les conduites individuelles et collectives. Elles sont rivées aux discours en tant que pratiques, en tant que systèmes symboliques, en tant que productions et reproductions des réalités sociales. Leurs fonctions cognitives, opératives et figuratives, explicatrices et implicatrices, commencent à être bien connues. Leurs fonctions symboliques, par contre, restent encore dissimulées sous les espèces des théories du capital symbolique/marché des biens symboliques, dans les plis de la doctrine des simulacres ou dans la philosophie des formes symboliques.

Les recherches de Piaget sur la formation des symboles montrent que les représentations naissent de l'union de signifiants, d'une connexion spécifique entre des signifiants et des signifiés, désormais différenciés. Il s'agit de la fonction symbolique générale qui est à l'origine de l'acquisition du langage et des signes collectifs. Par elle et grâce à elle, l'expérience en tant qu'action et construction 
progressives trouve ses propres conditions sociales d'existence; par elle et grâce à elle tous les système de représentations, langagiers ou autres, les arguments et les repères cognitifs, peuvent désigner des classes d'actions ou d'objet, donc produire des significations individuelles et sociales. Les sciences sociales se situent au cœur même de tout cela. Elles se placent, en effet, dans le processus de production et de reproduction des représentations, et elles se présentent à nous tout d'abord comme discours, comme activité verbale. Il va de soi que le discours social, par-delà l'échange et la confrontation, la neutralité et l'objectivité qu'il affiche et proclame, demeure la manifestation de rapports de force et parfois même il est le support de l'exercice du pouvoir. Dès lors, il est important de connaître les normes du discours des disciplines des sciences sociales. Elles sont les mêmes que celles de la pensée argumentative: normes d'une activité qui se cherche et se préoccupe d'actions locales; normes d'une activités orientée et finalisée par des intérêts et/ou par des valorisations; normes d'une activité proposée et opposée à d'autres activités dans une situation donnée; normes d'une activité qui ne vise ni le vrai ni l'universelle, mais le vraisemblable, le plausible, l'efficace. Si les constructions des sciences sociales sont produites par ces normes et sont la prise de conscience, la généralisation d'actes pour ainsi dire quotidiens, nous pouvons, donc, les lire pour connaître ce qu'elles disent, pour en voir la consistance, bref pour connaître la «logique» rendant possible et plausible la production du discours social. Dans ce même discours on peut aussi rechercher des indices nous permettant de comprendre les mécanismes de fonctionnement, dans les situations historiques et sociales, de la raison symboliques et signifiante, c'est-à-dire comment s'élaborent et se règlent, de cas en cas, les représentations dans les situations d'interaction comme dans celle d'intervention.

Jean Blaise Grize, dont les recherches novatrices ont changé ma façon de voir la sociologie, est convaincu que nous schématisons l'information lorsque nous construisons un ensemble signifiant, dans le sens que ce qui est dit reste inséparable de sa mise en forme. La «schématisation», selon Grize, révèle comment le discours appréhende la situation dans laquelle il se produit, la manière selon laquelle il est structuré en fonction d'une perspective donnée, de même que les réglages effectués en cours de construction et de transformation ${ }^{80}$.

Nous retrouvons aisément ce processus de schématisation dans toutes les théories des sciences sociales, avec le cortège des opérations de détermination, comme la constitution d'objet, la prédication, la mise en relation, et en outre des opérations d'acceptabilité, de cohésion, etc. Comme toute argumentation, les théories sociales s'adressent à un auditoire. Elles impliquent, par conséquent, des interventions acceptables, des opérations d'éclairage plus ou moins en rapport avec des valeurs, des idéologies, voire les sagesses; elles présupposent un consensus et des compétences liés aux situations dans lesquelles elles se produisent, et en fonction d'une perspective finalisée. Tout cela explique pourquoi il n'y a pas de

so J.-B. Grize, De la logique à l'argumentation. Préface de G. Busino, Genève, Droz, 1982; Logique et langage, Paris, Ophrys, 1990; Logique naturelle et communications, Paris, Puf, 1996; Les deux faces de l'argumentation. L'inférence et la déduction, in L'argumentation. Preuve et persuasion, Paris, Ed. de l'EHESS, 2002, pp. 13-27. Dans ce dernier volume, l'article de P. Livet, Formaliser l'argumentation en restant sensible au contexte, pp. 49-66, ouvre une perspective très intéressante et qu'il faut continuer à explorer. 
théorie unifiée et englobante; pourquoi il n'y a pas de solutions définitives aux problèmes sociaux et à ceux de l'organisation sociale; pourquoi les questions sociales changent ou resurgissent au gré du hasard des schématisations, des points de vue et des éclairages différents; pourquoi les concepts et les notions des sciences sociales sont la plupart du temps métaphoriques; pourquoi ces disciplines empruntent souvent à d'autres disciplines, en les utilisant ensuite analogiquement, les catégories d'identification et de référenciation.

Précisément pour toutes ces raisons il n'y a jamais eu, il n'y a pas en sciences sociales de correspondance biunivoque entre les faits et les propositions théoriques; pour ces raisons également, nous formulons des propositions non équivalentes selon les régions et les époques, selon les situations et les conditions particulières et spécifiques. Il est pratiquement impossible de falsifier une théorie sociale. Il suffit de penser à la théorie du suicide de Durkheim ou à celle de la bureaucratie de Max Weber ou à celle de la circulation des élites de Pareto. Il est courant, par contre, que des théories formellement fausses soient significativement signifiantes et manifestent, parfois, des apparences d'application. La vérité d'une théorie sociale n'est guère vérifiable, tandis que son utilité sociale, au demeurant variable, peut être facilement observée.

Liées aux activités pratico-cognitives des hommes et des groupes sociaux, guidées par la raison délibérative et animées par la pensée argumentative, nos théories sociales, changeantes et provisoires, engendrent des «faits» mais ne correspondent jamais à des «faits», ne sont jamais validées que par des «faits» produits par elles-mêmes. Les sciences sociales sont prisonnières d'un univers sémantique dont la clôture est insurmontable.

Comment faire correspondre une hypothèse aux faits alors que nous sommes dans l'impossibilité absolue de préciser préalablement quand telle ou telle autre correspondance aura lieu ou bien quand nous nous approcherons de l'une d'elles? Il s'agit là d'une impossibilité absolue puisque la conjecture au sujet de ces faits a la même signification que l'hypothèse qui parle de ces mêmes faits. Comment négliger que le langage baigne dans de la signification et que nous comprenons ce qu'on nous dit pas du tout à partir des règles formelles du dit langage?

Les sciences sociales sont-elles les sciences des rapports sociaux, rapports gouvernés par les logiques brutes et naturelles, ou par d'autres espèces de logiques? Sont-elles des sciences fondées sur des modes de rationalité différents de ceux qu'exige la raison abstraite de notre tradition culturelle dominante? Sontelles les sciences des autres raisons, des autres logiques, par exemple celles des sentiments?

Les réponses à ces questions sont un bric-à-brac de pièces à conviction. Cependant l'histoire des sciences permet d'affirmer qu'il y a des pluralités de critères pour établir la scientificité d'une discipline, et ceci est valable aussi pour les sciences de l'homme et de la société. Jean Ladrière a démontré que les critères de scientificité admissibles sont ceux que gouverne une solide capacité auto-organisatrice. Jean-Blaise Grize, de son côté, appelle cette capacité «schématisation » et croit entrevoir dans toutes les connaissances dicibles et transmissibles d'autres raisons, des raisons dissemblables de celles de la pensée logicomathématique. Passeron affirme que la succession et la juxtaposition des observations réglées sont à l'origine d'effets d'intelligibilité (l'effet propre produit sur chaque énoncé par son appartenance au langage organisé de l'énonciation). La connaissance 
sociologique est basée sur des liens logiques entre les opérations de constat et les formulations du discours des sociologues. Quant à moi, je crois qu'il s'agit d'hypothèses de travail très intéressantes, qu'il est nécessaire d'approfondir sans négliger toutefois deux faits capitaux: les produits du travail d'enquête ne se stabilisent jamais en théories au sens strict et le sens des énoncés théoriques reste plus ou moins redevable au sens des énoncés produits spontanément par les pratiques sociales. Chaque contexte impose ses règles, ses conventions, désigne ses destinataires, suppose des attentes particulières, révèle que les pensées et les conduites individuelles ne sont pas structurées par une structure mentale unique, qu'il y a une pluralité de systèmes de croyances, de modes de raisonnements, de styles de rationalité, de catégories qualifiant les genres, les formes et les statuts des discours et des énoncés.

La crise de la raison dont on parle depuis plusieurs années, est la crise d'une raison mais c'est également l'annonce de la découverte d'autres raisons. Il y a là également l'annonce de la fin des querelles sur la nature, scientifique ou non, des sciences humaines et sociales. Et peut-être la reconnaissance de la pleine dignité scientifique des sciences de l'homme et de la société, instruments indispensables pour construire des représentations, pour donner du sens, pour attribuer des significations, pour donner une existence signifiante à la vie des individus et à la vie sociale des groupes.

Genève 